\title{
Optimal dividend and reinsurance strategies with financing and liquidation value
}

\author{
Dingjun Yao ${ }^{\mathrm{a}, \mathrm{b}}$, Hailiang Yang ${ }^{\mathrm{c}, *}$, Rongming Wang ${ }^{\mathrm{d}}$ \\ ${ }^{a}$ School of Finance, Nanjing University of Finance and Economics, Nanjing 210023, China \\ ${ }^{b}$ The Center of Cooperative Innovation for Modern Service Industry, Nanjing 210023, China \\ ${ }^{c}$ Department of Statistics and Actuarial Science, The University of Hong Kong, Hong Kong, China \\ ${ }^{d}$ School of Statistics, East China Normal University, Shanghai 200241, China
}

\begin{abstract}
This study investigates a combined optimal financing, reinsurance and dividend distribution problem for a big insurance portfolio. A manager can control the surplus by buying proportional reinsurance, paying dividends and raising money dynamically. The transaction costs and liquidation values at bankruptcy are included in the risk model. Under the objective of maximising the insurance company's value, we identify the insurer's joint optimal strategies using stochastic control methods. The results reveal that managers should consider financing if and only if the terminal value and the transaction costs are not too high, less reinsurance is bought when the surplus increases or dividends are always distributed using the barrier strategy.
\end{abstract}

Keywords: Dividend strategy; Financing strategy; Proportional reinsurance; Transaction costs; Liquidation value

\section{Introduction}

In the actuarial and mathematical insurance literature, the classical dividend problem consists of finding the dividend distribution policy that maximises the expected total discounted dividend payments until the time of bankruptcy. However, more complicated models involve controlling both the risk-related activities and the dividend distribution. This approach becomes particularly important when modelling the behaviour of a large insurance company. Frequently, in addition to the distribution of part of the surplus as dividends, an insurance company's manager faces the problem of how much risk must be ceded by purchasing reinsurance. Reinsurance refers to controlling revenues by diverting a portion of premiums to a reinsurer to reduce the insurer's risk, which also reduces the insurer's potential profit. A reinsurance contract is said to be "cheap" if the cedent pays the same fraction of the premium as the reinsured. In a "non-cheap" reinsurance contract, the cedent pays a larger fraction of the premium than the fraction to be reinsured. The excess can be interpreted as the transaction cost for a reinsurance contract. Recently, some attention has been paid to the combined optimal dividend and reinsurance problem for risk models. As an extension of the classical dividend problem, it assumes that the manager of an insurance company can control the dividend stream and risk exposure in terms of reinsurance. Proportional and excess-of-loss reinsurance have received increasing attention from academics and practitioners. The literature on the combined optimisation of dividend distribution and proportional reinsurance includes Taksar and Zhou (1998), Hogaard and Taksar (1999, 2004), Taksar (2000a), Choulli et al. (2003), Cadenillas et al. (2006) and Chen et al. (2013). Studies on the combined optimisation of dividend distribution and excess-of-loss reinsurance include Asmussen et al. (2000), Mnif and Sulem (2005), Bai et al. (2010), Liu and $\mathrm{Hu}(2014)$ and the references therein.

When a company is on the verge of bankruptcy, it faces two choices: be bailed out through financing, or get out of the business. A bail-out requires financing costs, such as the proportional and fixed transaction costs generated by the advisory, consulting and issuance of securities. Leaving the business may lead to bankruptcy and the corresponding liquidation (or terminal) value, say $P$. The liquidation value can be viewed as the salvage value for $P \geq 0$ and the penalty amount for $P<0$. The decision to raise money or not depends on the relationships among the model's parameters. To maximise the company's value, the manager must seek optimal financing, reinsurance and dividend distribution strategies. Most of the studies in the literature deal with this optimisation problem in the case of $P=0$. The company's value is measured by the expected discounted total dividends minus the expected discounted costs of financing until the time of bankruptcy. For example, He

\footnotetext{
*Corresponding author. Email: hlyang@hku.hk
} 
and Liang (2009) and Barth and Moreno-Bromberg (2014) studied the dividend and financing problem with "cheap" proportional reinsurance. Peng et al. (2012) further generalised the optimisation problem for a case of "non-cheap" proportional reinsurance, but they assumed that the bankruptcy never occurred based on the belief that financing would be applied when needed. Guan and Liang (2014) extended these risk models by allowing for "non-cheap" reinsurance and considering the possibility of bankruptcy. The proportional and fixed costs in financing and dividend distribution processes have also been considered. For more detailed discussions on this issue, see, for example, Meng and Siu (2011), Zhou and Yuen (2012) and the references therein. Generally speaking, non-zero liquidation better reflects reality, but it also complicates the optimisation problem. A few studies have investigated the optimal dividend and reinsurance problem with non-zero liquidation, but without financing. They have measured the company's value by the sum of the expected present value of all dividends until bankruptcy and the expected discounted liquidation value. For example, Taksar (2000b) and Xu and Zhou (2012) studied the optimal dividend problems assuming "cheap" proportional and excess-of-loss reinsurance, respectively, in diffusion models with a liquidation value $P \geq 0$. Note that in the case of "cheap" reinsurance, the insurer can avoid debt liability by ceding all risks. Theoretically, the insurer can keep the surplus nonnegative forever by purchasing reinsurance. It is always optimal to avoid bankruptcy for the insurer if the liquidation value is negative. Thus, the case of $P<0$ becomes trivial. Taksar and Hunderup (2007) and Yao et al. (2014) encountered similar situations. Clearly, this conclusion does not agree with the practice. Sometimes, negative liquidation value is unavoidable, which drives the exploration of the problem under the assumptions of "non-cheap" proportional reinsurance and arbitrary liquidation value. To the best of our knowledge, Liang and Young (2012) first investigated this problem with arbitrary liquidation value $P \in \mathbb{R}$ when one controls both the dividend distribution and the "non-cheap" proportional reinsurance. Optimal dividend and reinsurance strategies were obtained using the Legendre transform. They did not consider financing and transaction costs. Yao et al. (2014) first focused on the combined optimisation problem of financing, reinsurance and dividend distribution with positive liquidation value. They assumed that the premium was calculated via the variance principle, and thus analysed the effects of proportional and fixed transaction costs. With the exception of Yao et al. (2014), very little work has considered the combined optimal financing, dividend and reinsurance strategies with non-zero liquidation value.

Motivated by the above references, in this study we examine an optimal financing, dividend and "noncheap" reinsurance problem with arbitrary liquidation value. We include transaction costs in our risk model. Our objective is to find the optimal management strategies for maximising company value, which is measured by a new reasonable performance function. We extend the risk model in Liang and Young (2012) by taking financing and transaction costs into account and using techniques beyond the Legendre transform to solve the problem. Then, we provide explicit solutions for the value function and the optimal strategy in 14 different cases and analyse the influence of transaction costs and liquidation value $P \in \mathbb{R}$. The remainder of this paper is organised as follows. In Section 2, we use a diffusion approximation of the Cramér-Lundberg model with reinsurance to formulate the optimisation problem for a controlled diffusion model with dividend, financing and "non-cheap" reinsurance policies. In Section 3, the Hamilton-Jacobi-Bellman (HJB) equations associated with the optimisation problem are given and some of the properties of the value function are discussed. Based on the costs of reinsurance, as measured by the safety loading, we address the solutions to the value function and associated optimal strategy in Sections 4 and 5, respectively. Section 6 concludes the study.

\section{Model formulation and the optimal control problem}

We start with the classical Cramér-Lundberg risk model. In this model, claims arrive according to a Poisson process $N_{t}$ with a rate of $\lambda$ and the size of the $i$ th claim is $Y_{i}$, where $Y_{i}$ 's are independent and identically distributed. Assume that the mean $\mu_{1}=\mathrm{E}\left(Y_{1}\right)$ and the second moment $\mu_{2}=\mathrm{E}\left(Y_{1}^{2}\right)$ are finite. The risk process representing the company's surplus evolves according to

$$
U_{t}=x+c t-\sum_{i=1}^{N_{t}} Y_{i}
$$

where $U_{0}=x \geq 0$ is the initial surplus and $c>0$ is the premium rate. Under the assumption of the expected value principle, it has

$$
c=\left(1+\theta_{1}\right) \mathrm{E}\left(\sum_{i=1}^{N_{1}} Y_{i}\right)=\left(1+\theta_{1}\right) \lambda \mu_{1},
$$


where $\theta_{1}>0$ is the safety loading for the insurer. Suppose that the insurer purchases a proportional reinsurance with a retention level of $q \in[0,1]$. Specifically, for each claim of size $Y_{i}$, the insurer covers $q Y_{i}$ and the reinsurer covers the rest $(1-q) Y_{i}$. Suppose that the reinsurer also uses the expected premium principle, but with a larger safety loading $\theta_{2} \in\left(\theta_{1}, \infty\right)$, i.e., the reinsurance is "non-cheap", then the premium rate for reinsurance is

$$
c^{q}=\left(1+\theta_{2}\right) \mathrm{E}\left(\sum_{i=1}^{N_{1}}(1-q) Y_{i}\right)=\left(1+\theta_{2}\right)(1-q) \lambda \mu_{1} .
$$

Then, the surplus process with reinsurance can be expressed as

$$
U_{t}^{q}=x+\left(c-c^{q}\right) t-\sum_{i=1}^{N_{t}} q Y_{i},
$$

where $U_{0}^{q}=x \geq 0$. Let $\left(\Omega, \mathcal{F},\left\{\mathcal{F}_{t}\right\}_{t \geq 0}, \mathrm{P}\right)$ be a probability space, where $\left\{\mathcal{F}_{t}\right\}_{t \geq 0}$ is an information filtration, and let $\left\{B_{t}\right\}_{t \geq 0}$ be a standard Brownian motion adapted to $\mathcal{F}_{t}$. According to Grandell (1991), we approximate model (2.4) by a pure diffusion model $\left\{X_{t}^{q}\right\}_{t \geq 0}$ with the same drift and volatility; that is, $X_{t}^{q}$ satisfies the following stochastic process

$$
X_{t}^{q}=x+a\left(\theta_{1}-(1-q) \theta_{2}\right) t+b q B_{t},
$$

where $X_{0}^{q}=x, a=\lambda \mu_{1}$ and $b=\sqrt{\lambda \mu_{2}}$. Such an approximation is suitable for large portfolios.

Suppose that $q \in[0,1]$ can be adjusted dynamically to control the risk exposure, then we use the process $\left\{q_{t}\right\}_{t \geq 0}$ to describe a reinsurance strategy. In addition, we incorporate dividend distribution and financing in model (2.5). Let $D_{t}$ denote the total amount of dividends paid from time 0 to $t$. Let $R_{t}=\sum_{i=1}^{\infty} I_{\left\{\tau_{i} \leq t\right\}} \eta_{i}$ denote the total amount of capital raised by issuing equities from time 0 to $t$, where $\left\{\tau_{i}, i=1,2, \cdots\right\}$ denote the time points when the equity is issued and $\left\{\eta_{i}, i=1,2, \cdots\right\}$ denote the amounts of equity issued. When applying strategy $\pi=\left(q^{\pi}, D^{\pi}, R^{\pi}\right)$, the resulting surplus process is modelled by

$$
X_{t}^{\pi}=x+\int_{0}^{t} a\left(\theta_{1}-\left(1-q_{s}^{\pi}\right) \theta_{2}\right) \mathrm{d} s+\int_{0}^{t} b q_{s}^{\pi} \mathrm{d} B_{s}-D_{t}^{\pi}+R_{t}^{\pi},
$$

where $X_{0}^{\pi}=x$. The definition of an admissible strategy that can be selected by the manager is as follows.

Definition 2.1. A strategy $\pi=\left(q^{\pi}, D^{\pi}, R^{\pi}\right)$ is said to be admissible if it satisfies the following conditions:

(i) The retention level $q^{\pi}=\left\{q_{t}^{\pi}\right\}_{t \geq 0}$ is an $\mathcal{F}_{t^{-}}$adapted process with $0 \leq q_{t}^{\pi} \leq 1$ for all $t \geq 0$.

(ii) $\left\{D_{t}^{\pi}\right\}$ is an increasing, $\mathcal{F}_{t}$-adapted càdlàg process with $D_{0-}^{\pi}=0$ and satisfies $\Delta D_{t}^{\pi}=D_{t}^{\pi}-D_{t-}^{\pi} \leq X_{t-}^{\pi}$ for all $t \geq 0$.

(iii) $\left\{\tau_{i}^{\pi}\right\}$ is a sequence of stopping times w.r.t. $\mathcal{F}_{t}$ and $0 \leq \tau_{1}^{\pi}<\cdots<\tau_{i}^{\pi}<\cdots$, a.s..

(iv) $\eta_{i}^{\pi} \geq 0, i=1,2, \cdots$ is measurable w.r.t. $\mathcal{F}_{\tau_{i}^{\pi}}$.

(v) $\mathrm{P}\left(\lim _{i \rightarrow \infty} \tau_{i}^{\pi}<t\right)=0, \forall t>0$.

Condition (ii) means that the total amount of dividends is less than the surplus available at that time. Condition $(v)$ implies that the issuance of equities may not occur infinitely in a finite time interval. We write $\Pi$ for the space of these admissible strategies. For each $\pi \in \Pi$, the bankruptcy time is defined as $T^{\pi}=\inf \left\{t \geq 0: X_{t}^{\pi}<0\right\}$, which is the first time that the surplus becomes negative.

Problem 2.1. We measure the company's value associated with strategy $\pi \in \Pi$ using the following performance function:

$$
V(x ; \pi)=\mathrm{E}_{x}\left(\beta_{1} \int_{0}^{T^{\pi}} e^{-\delta s} \mathrm{~d} D_{s}^{\pi}+P e^{-\delta T^{\pi}}-\sum_{n=1}^{\infty} e^{-\delta \tau_{n}^{\pi}}\left(\beta_{2} \eta_{n}^{\pi}+K\right) I_{\left\{\tau_{n}^{\pi} \leq T^{\pi}\right\}}\right),
$$

which is the expected sum of the discounted liquidation value and the discounted dividends less the expected discounted costs of equity issuances until the time of bankruptcy. $\mathrm{E}_{x}$ denotes the expectation conditional on $X_{0}^{\pi}=x$ and $\delta>0$ is the discount factor. In the dividend distribution process, $\beta_{1} \in(0,1)$ is the proportional transaction cost factor, which means that the shareholders can get $\beta_{1} l$ if the company pays $l$ as dividends. In the financing process, $\beta_{2}>1$ is the proportional transaction cost factor and $K>0$ is the fixed cost, such that the shareholders need to pay $\beta_{2} \eta+K$ to meet the capital injection of $\eta$. We are interested in finding the value function

$$
V(x)=\max _{\pi \in \Pi} V(x ; \pi)
$$

and the associated optimal strategy $\pi^{*}$, such that $V(x)=V\left(x ; \pi^{*}\right)$. 


\section{HJB equation and preliminary analysis}

Suppose that $v:[0, \infty) \mapsto \mathbb{R}$ is a candidate solution for the value function. Let $\mathscr{C}$ denote the financing operator defined by $\mathscr{C} v(x)=\sup _{y \geq 0}\left\{v(x+y)-\beta_{2} y-K\right\}$, which represents the value of the strategy that consists of choosing the best immediate equity issuance. Another notation used in this paper is the differential operator $\mathscr{A}^{q}$, defined by $\mathscr{A}^{q} v(x)=\frac{1}{2} q^{2} b^{2} v^{\prime \prime}(x)+\left(\theta_{1}-(1-q) \theta_{2}\right) a v^{\prime}(x)-\delta v(x)$.

Assume that $v(x)$ is sufficiently smooth and regular to perform the following manipulations. Then, if the process starts at $x \geq 0$ and follows an optimal strategy, the performance function associated with this optimal strategy is $v(x)$. In contrast, if the process starts at $x$, selects the best immediate equity issuance and then follows an optimal strategy, then the performance function associated with this second strategy is $\mathscr{C} v(x)$. Given that the first strategy is optimal, its performance function is larger than that associated with the second strategy. Furthermore, these two performance functions are equivalent when it is optimal to finance. Hence, $v(x) \geq \mathscr{C} v(x)$. In the continuation region, that is, when the manager does not intervene, we must have $\mathscr{A}^{q} v(x)=0$. In the dividend region, we must have $v^{\prime}(x)=\beta_{1}$. Considering the Markovian structure of the problem, as the insurance company is on the brink of bankruptcy, the optimal strategy should either allow for the surplus process to hit $(-\infty, 0)$ by issuing no new equity, which corresponds to the boundary condition $v(0)=P$ and $\mathscr{C} v(0) \leq v(0)$, or keep the surplus process in the interval $[0, \infty)$, which corresponds to the boundary condition $v(0) \geq P$ and $\mathscr{C} v(0)=v(0)$. Using stochastic control theory, see Fleming and Soner (1993), we write the HJB equations associated with Problem 2.1 as

$$
\begin{aligned}
& \max \left\{\mathscr{C} v(x)-v(x), \max _{0 \leq q \leq 1}\left\{\mathscr{A}^{q} v(x)\right\}, \beta_{1}-v^{\prime}(x)\right\}=0 \\
& \max \{\mathscr{C} v(0)-v(0), P-v(0)\}=0 .
\end{aligned}
$$

Theorem 3.1. Suppose that $v(x)$ is an increasing, concave and twice continuously differentiable solution to HJB equations (3.1) and (3.2), and that the derivative $v^{\prime}(x)$ is bounded, then $v(x) \geq V(x ; \pi)$ for any admissible strategy $\pi \in \Pi$, such that $v(x) \geq V(x)$. Furthermore, if there exists some strategy $\pi^{*}=\left(q^{\pi^{*}}, D^{\pi^{*}}, R^{\pi^{*}}\right) \in \Pi$, such that $v(x)=V\left(x ; \pi^{*}\right)$, then $v(x)=V(x)$ and $\pi^{*}$ is optimal.

Proof. See Appendix A.

The above theorem drives us to find an appropriate solution to the HJB equations and construct the associated optimal strategy $\pi^{*}$. In addition, given the time value of money, it is optimal to postpone raising money for as long as possible, i.e., issuance of equity may happen when and only when the surplus process hits the barrier 0 . The result can be established by repeating a procedure that is similar to that in Lemma 3.2 of Peng et al. (2012). Thus, $\mathscr{C} v(x)-v(x)<0$ holds for all $x>0$.

Lemma 3.1. The value function $V(x)$ defined by (2.8) is increasing for $x \geq 0$ with

$$
\beta_{1}(x-y) \leq V(x)-V(y) \leq \beta_{2}(x-y)+K
$$

and satisfies the following bounded condition

$$
\beta_{1} x+P \leq V(x) \leq \beta_{1} x+\beta_{1} \theta_{1} a / \delta+P I_{\{P>0\}} .
$$

Proof. Consider an admissible strategy $\pi_{1}$ with $V\left(x ; \pi_{1}\right) \geq V(x)-\varepsilon$ for any $\varepsilon>0$. For $y<x$, we define a new admissible strategy as follows. Collect $x-y$ dollars by issuing equities immediately and then take the strategy $\pi_{1}$ with initial capital $x$. Then, for $\varepsilon>0$, we have

$$
V(y) \geq V\left(x ; \pi_{1}\right)-\beta_{2}(x-y)-K \geq V(x)-\varepsilon-\beta_{2}(x-y)-K .
$$

Because $\varepsilon$ is arbitrary, $V(x)-V(y) \leq \beta_{2}(x-y)-K$. The first inequality in (3.3) can be similarly proven.

We now consider another admissible strategy $\pi_{2}$. Simultaneously distribute all of the surplus as dividends and claim the liquidation value. Then, the corresponding performance function is $V\left(x ; \pi_{2}\right)=\beta_{1} x+P$. Due to the optimality of the value function, we have $V(x) \geq V\left(x ; \pi_{2}\right)=\beta_{1} x+P$.

Recall the surplus process $X_{t}^{q}$ with only reinsurance in (2.5). We know

$$
\mathrm{E}_{x}\left(\int_{0}^{T^{\pi}} e^{-\delta s} \mathrm{~d} X_{s}^{q}\right)=\mathrm{E}_{x}\left(\int_{0}^{T^{\pi}} e^{-\delta s}\left(a\left(\theta_{1}-(1-q) \theta_{2}\right)\right) \mathrm{d} s\right) \leq \theta_{1} a / \delta .
$$


By Itô's formula,

$$
e^{-\delta T^{\pi}} X_{T^{\pi}}^{\pi}=x-\delta \int_{0}^{T^{\pi}} e^{-\delta s} X_{s}^{\pi} \mathrm{d} s+\int_{0}^{T^{\pi}} e^{-\delta s} \mathrm{~d} X_{s}^{\pi} .
$$

Given that $X_{T^{\pi}}^{\pi}=0$ and $X_{t}^{\pi} \geq 0$, for $t \leq T^{\pi}$, taking the expectation on both sides yields

$$
-\mathrm{E}_{x}\left(\int_{0}^{T^{\pi}} e^{-\delta s} \mathrm{~d} X_{s}^{\pi}\right)=x-\mathrm{E}_{x}\left(\delta \int_{0}^{T^{\pi}} e^{-\delta s} X_{s}^{\pi} \mathrm{d} s\right) \leq x .
$$

Then,

$$
\begin{aligned}
V(x ; \pi) & =\mathrm{E}_{x}\left(\beta_{1} \int_{0}^{T^{\pi}} e^{-\delta s} \mathrm{~d} D_{s}^{\pi}+P e^{-\delta T^{\pi}}-\sum_{n=1}^{\infty} e^{-\delta \tau_{n}^{\pi}}\left(\beta_{2} \eta_{n}^{\pi}+K\right) I_{\left\{\tau_{n}^{\pi} \leq T^{\pi}\right\}}\right) \\
& \leq \beta_{1} \mathrm{E}_{x}\left(\int_{0}^{T^{\pi}} e^{-\delta s} \mathrm{~d} D_{s}^{\pi}-\sum_{n=1}^{\infty} e^{-\delta \tau_{n}^{\pi}} \eta_{n}^{\pi} I_{\left\{\tau_{n}^{\pi} \leq T^{\pi}\right\}}\right)+P I_{\{P>0\}} \\
& =\beta_{1}\left[\mathrm{E}_{x}\left(\int_{0}^{T^{\pi}} e^{-\delta s} \mathrm{~d} X_{s}^{q}\right)-\mathrm{E}_{x}\left(\int_{0}^{T^{\pi}} e^{-\delta s} \mathrm{~d} X_{s}^{\pi}\right)\right]+P I_{\{P>0\}} \\
& \leq \beta_{1} x+\beta_{1} \theta_{1} a / \delta+P I_{\{P>0\}} .
\end{aligned}
$$

Hence, the result follows.

Reinsurance can reduce the insurer's risk and potential profit. It is understandable that the insurer should buy less reinsurance as its cost increases, which is measured by the safety loading $\theta_{2}$ for the reinsurer. It is expected that full retention will be taken once $\theta_{2}$ exceeds some critical level. In the following, we split the optimisation problem into two parts according to the critical level $\theta_{1}+\sqrt{\theta_{1}^{2}+2 \delta\left(\frac{b}{a}\right)^{2}}$.

\section{The case of $\theta_{2} \in\left(\theta_{1}, \theta_{1}+\sqrt{\theta_{1}^{2}+2 \delta\left(\frac{b}{a}\right)^{2}}\right)$}

Throughout this section, we only consider the case when the safety loading for the reinsurer satisfies

$$
\theta_{1}<\theta_{2}<\theta_{1}+\sqrt{\theta_{1}^{2}+2 \delta\left(\frac{b}{a}\right)^{2}} .
$$

Motivated by the innovative ideas in L $\phi \mathrm{kka}$ and Zervos (2008), we discuss the solutions to HJB equations according to different conditions, such that (3.2) holds. In addition, we expect the solution to be increasing, concave and twice continuously differentiable, and for the derivative to be bounded.

\subsection{The case without financing}

We now consider the first case with $v(0)=P$ and $\mathscr{C} v(0)-v(0) \leq 0$. It is optimal to declare bankruptcy whenever the surplus is zero and financing is unprofitable. Using methodologies from stochastic control theory, in this case the solution $f(x)$ for $v(x)$ should satisfy

$$
\begin{aligned}
& \max _{0 \leq q \leq 1}\left\{\mathscr{A}^{q} f(x)\right\}=0,0<x \leq u_{0}, \\
& \beta_{1}-f^{\prime}(x)=0, \quad x \geq u_{0}, \\
& f(0)=P \\
& \mathscr{C} f(0)-f(0) \leq 0
\end{aligned}
$$

where unknown parameters $0 \leq u_{0}<\infty$. In fact, (4.2)-(4.5) mean that the continuation region is $\left[0, u_{0}\right]$, the dividend region is $\left[u_{0}, \infty\right)$ and the financing region is empty. Differentiating (4.2) with respect to $q$ and setting the derivative equal to zero yields

$$
q(x)=\frac{-\theta_{2} a f^{\prime}(x)}{b^{2} f^{\prime \prime}(x)}, \quad \text { if } f^{\prime \prime}(x) \neq 0 .
$$


Plugging (4.6) into (4.2) yields

$$
\left(\frac{1}{2} \theta_{2} a q+\left(\theta_{1}-\theta_{2}\right) a\right) f^{\prime}(x)-\delta f(x)=0 .
$$

Note that $q$ is a function of $x$. By taking the derivative with respect to $x$ on both sides of (4.7) and using (4.6) again, we find

$$
q^{\prime}(x)=\frac{\left(2 \delta b^{2}+a^{2} \theta_{2}^{2}\right) q(x)-2 \theta_{2}\left(\theta_{2}-\theta_{1}\right) a^{2}}{\theta_{2} a b^{2} q(x)} .
$$

To proceed with the analysis, we define an increasing linear function on $[0, \infty)$

$$
\phi(x):=\left(2 \delta b^{2}+a^{2} \theta_{2}^{2}\right) x-2 \theta_{2}\left(\theta_{2}-\theta_{1}\right) a^{2} .
$$

Under condition (4.1), there is a unique solution

$$
\rho=\frac{2 \theta_{2}\left(\theta_{2}-\theta_{1}\right) a^{2}}{2 \delta b^{2}+a^{2} \theta_{2}^{2}} \in(0,1)
$$

where $\phi(\rho)=0$. Clearly, $\phi(x)>0$ for all $x \in(\rho, \infty)$. Assuming that $q(0):=q_{0} \in(\rho, 1]$, we define another function of $x \in\left[q_{0}, 1\right]$ as

$$
Q(x)=\int_{q_{0}}^{x} \frac{\theta_{2} a b^{2} y}{\left(2 \delta b^{2}+a^{2} \theta_{2}^{2}\right) y-2 \theta_{2}\left(\theta_{2}-\theta_{1}\right) a^{2}} \mathrm{~d} y .
$$

Due to the positivity of the integrand, $Q(x)$ is strictly increasing and $Q(1)<\infty$. Consequently, the inverse $Q^{-1}(x)$ of the function $Q(x)$ exists on $\left[0, x_{0}\right]$ with $x_{0}=Q(1)$. We conjecture that the switch level for reinsurance $x_{0}$ is smaller than that for dividend $u_{0}$. Together with the condition $Q\left(q_{0}\right)=0$, we have

$$
q(x)=Q^{-1}(x), \quad 0 \leq x \leq x_{0} .
$$

Given (4.4) and (4.6), we can express $f(x)$ through $q(x)$ by

$$
f(x)=k_{3} \int_{0}^{x} e^{\int_{y}^{x_{0}} \frac{\theta_{2} a}{b^{2} q(z)} \mathrm{d} z} \mathrm{~d} y+P,
$$

where $k_{3}>0$ needs to be determined.

Regarding the continuity of retention level, we conjecture that the insurer will take all risks when the surplus exceeds $x_{0}$; that is, $q(x) \equiv 1$ for $x \in\left[x_{0}, u_{0}\right]$. Then, (4.2) becomes a second-order ordinary differential equation

$$
\frac{1}{2} b^{2} f^{\prime \prime}(x)+\theta_{1} a f^{\prime}(x)-\delta f(x)=0 .
$$

Its solution is of the form

$$
f(x)=k_{1} e^{r_{+}\left(x-x_{0}\right)}+k_{2} e^{r_{-}\left(x-x_{0}\right)},
$$

with undetermined coefficients $k_{1}$ and $k_{2}$ and

$$
\begin{aligned}
& r_{+}=\frac{a}{b^{2}}\left(-\theta_{1}+\sqrt{\theta_{1}^{2}+2 \delta\left(\frac{b}{a}\right)^{2}}\right)>0, \\
& r_{-}=\frac{a}{b^{2}}\left(-\theta_{1}-\sqrt{\theta_{1}^{2}+2 \delta\left(\frac{b}{a}\right)^{2}}\right)<0 .
\end{aligned}
$$

Finally, combining (4.3) with the continuity of $f(x)$ yields

$$
f(x)=\beta_{1}\left(x-u_{0}\right)+f\left(u_{0}\right), \quad x \geq u_{0} .
$$

By setting the left first and second derivatives to equal the right first and second derivatives of $f(x)$ at $x_{0}$ and $u_{0}$, we obtain

$$
\begin{aligned}
& k_{1} r_{+}+k_{2} r_{-}=k_{3}, \\
& k_{1}\left(r_{+}\right)^{2}+k_{2}\left(r_{-}\right)^{2}=-\frac{\theta_{2} a}{b^{2}} k_{3}, \\
& k_{1} r_{+} e^{r_{+}\left(u_{0}-x_{0}\right)}+k_{2} r_{-} e^{r_{-}\left(u_{0}-x_{0}\right)}=\beta_{1}, \\
& k_{1}\left(r_{+}\right)^{2} e^{r_{+}\left(u_{0}-x_{0}\right)}+k_{2}\left(r_{-}\right)^{2} e^{r_{-}\left(u_{0}-x_{0}\right)}=0 .
\end{aligned}
$$


Solving (4.18) and (4.19) leads to

$$
k_{1}=k_{3} c_{1}, \quad k_{2}=k_{3} c_{2}
$$

where

$$
\begin{aligned}
& c_{1}=\frac{r_{-}+\frac{\theta_{2} a}{b^{2}}}{r_{+}\left(r_{-}-r_{+}\right)}>0, \\
& c_{2}=\frac{r_{+}+\frac{\theta_{2} a}{b^{2}}}{r_{-}\left(r_{+}-r_{-}\right)}<0 .
\end{aligned}
$$

Here, the inequality $c_{1}>0$ is valid due to (4.1), and $c_{2}<0$ is obvious. Substituting (4.22) into (4.21) yields

$$
u_{0}=x_{0}+\frac{1}{r_{+}-r_{-}} \ln \left(\frac{b^{2}+\frac{\theta_{2} a}{r_{+}}}{b^{2}+\frac{\theta_{2} a}{r_{-}}}\right)>x_{0},
$$

where the inequality holds because $b^{2}+\frac{\theta_{2} a}{r_{+}}>b^{2}+\frac{\theta_{2} a}{r_{-}}>0$ in the case of (4.1). Using (4.22) and (4.25), to solve (4.20) we have

$$
k_{3}=\frac{\beta_{1} b^{2}}{b^{2}+\frac{\theta_{2} a}{r_{+}}}\left(\frac{b^{2}+\frac{\theta_{2} a}{r_{+}}}{b^{2}+\frac{\theta_{2} a}{r_{-}}}\right)^{\frac{-r_{-}}{r_{+}-r_{-}}}>0 .
$$

Lemma 4.1. $\beta_{1}<k_{3}<\frac{\beta_{1} b^{2}}{b^{2}+\frac{\theta_{2} a}{r_{-}}}$and $\lim _{\theta_{2} \rightarrow-\frac{b^{2}}{a} r_{-}} k_{3}=\infty$.

Proof. To prove the left side of the inequality, we take the $\log$ of $k_{3}$. Due to the concavity of the $\log$ function, we obtain

$$
\begin{aligned}
\log k_{3} & =\log \beta_{1}+\log b^{2}-\left[\frac{r_{+}}{r_{+}-r_{-}} \log \left(b^{2}+\frac{\theta_{2} a}{r_{+}}\right)-\frac{r_{-}}{r_{+}-r_{-}} \log \left(b^{2}+\frac{\theta_{2} a}{r_{-}}\right)\right] \\
& >\log \beta_{1}+\log b^{2}-\log \left[\frac{r_{+}}{r_{+}-r_{-}}\left(b^{2}+\frac{\theta_{2} a}{r_{+}}\right)-\frac{r_{-}}{r_{+}-r_{-}}\left(b^{2}+\frac{\theta_{2} a}{r_{-}}\right)\right] \\
& =\log \beta_{1}+\log b^{2}-\log b^{2}=\log \beta_{1},
\end{aligned}
$$

which implies $k_{3}>\beta_{1}$. However, together with the following inequalities

$$
b^{2}+\frac{\theta_{2} a}{r_{+}}>b^{2}+\frac{\theta_{2} a}{r_{-}}>1, \quad 0<\frac{-r_{-}}{r_{+}-r_{-}}<1,
$$

we derive that

$$
k_{3}=\frac{\beta_{1} b^{2}}{b^{2}+\frac{\theta_{2} a}{r_{+}}}\left(\frac{b^{2}+\frac{\theta_{2} a}{r_{+}}}{b^{2}+\frac{\theta_{2} a}{r_{-}}}\right)^{\frac{-r_{-}}{r_{+}-r_{-}}}<\frac{\beta_{1} b^{2}}{b^{2}+\frac{\theta_{2} a}{r_{+}}} \cdot \frac{b^{2}+\frac{\theta_{2} a}{r_{+}}}{b^{2}+\frac{\theta_{2} a}{r_{-}}}=\frac{\beta_{1} b^{2}}{b^{2}+\frac{\theta_{2} a}{r_{-}}} .
$$

The inequality is confirmed. It is interesting to note that (4.1) can be re-expressed as $\theta_{1}<\theta_{2}<-\frac{b^{2}}{a} r_{-}$. It is also easy to see that

$$
\frac{\mathrm{d}}{\mathrm{d} \theta_{2}} \log k_{3}=\frac{-a^{2} \theta_{2}}{\left(b^{2} r_{+}+\theta_{2} a\right)\left(b^{2} r_{-}+\theta_{2} a\right)}>0 .
$$

Thus, $k_{3}:=k_{3}\left(\theta_{2}\right)$ is an increasing function on $\left(\theta_{1},-\frac{b^{2}}{a} r_{-}\right)$and $\lim _{\theta_{2} \rightarrow-\frac{b^{2}}{a} r_{-}} k_{3}=\infty$.

Thus far we obtain an increasing, concave and twice continuously differentiable solution to (4.2)-(4.4) as

$$
f(x)= \begin{cases}\beta_{1}\left(x-u_{0}\right)+f\left(u_{0}\right), & x \geq u_{0}, \\ k_{1} e^{r_{+}\left(x-x_{0}\right)}+k_{2} e^{r_{-}\left(x-x_{0}\right)}, & x_{0} \leq x \leq u_{0}, \\ k_{3} \int_{0}^{x} e^{\int_{y}^{x_{0}} \frac{\theta_{2} a}{b^{2} q(z)} \mathrm{d} z} \mathrm{~d} y+P, & 0 \leq x \leq x_{0},\end{cases}
$$


and an associated candidate optimal reinsurance policy characterised by

$$
q^{\pi^{*}}(x)= \begin{cases}1, & x \geq x_{0} \\ Q^{-1}(x), & 0 \leq x \leq x_{0}\end{cases}
$$

In what follows, we determine $q_{0}, x_{0}$ and $u_{0}$. From (4.7), we have

$$
k_{3}\left(\frac{1}{2} \theta_{2} a q_{0}+\theta_{1} a-\theta_{2} a\right) e^{\int_{0}^{x_{0}} \frac{\theta_{2} a}{b^{2} q(z)} \mathrm{d} z}=\delta P .
$$

By applying a variable change of $y=q(z)$ and combining it with (4.8), we arrive at

$$
P=\frac{k_{3}}{\delta} \Phi\left(q_{0}\right), \quad \rho<q_{0} \leq 1,
$$

where $\Phi(x):=\left(\frac{1}{2} \theta_{2} a x+\theta_{1} a-\theta_{2} a\right) e^{\int_{x}^{1} \frac{a^{2} \theta_{2}^{2}}{\phi(y)} \mathrm{d} y}$. Recalling $\phi(x)>0$ for $x \in(\rho, 1]$, we know that $\Phi(x)$ is strictly increasing on $(\rho, 1]$, as $\Phi^{\prime}(x)=\frac{a \delta \theta_{2} b^{2} x}{\phi(x)} e^{\int_{x}^{1} \frac{a^{2} \theta_{2}^{2}}{\phi(y)} \mathrm{d} y}>0$. So the maximum is $\Phi(1)=a\left(\theta_{1}-\frac{1}{2} \theta_{2}\right)$. In addition, we can rewrite $\frac{a^{2} \theta_{2}^{2}}{\phi(y)}:=\frac{\gamma}{y-\rho}$ with $\gamma=\frac{a^{2} \theta_{2}^{2}}{2 \delta b^{2}+a^{2} \theta_{2}^{2}}>0$. According to $\frac{1}{2} \theta_{2} a \rho+\theta_{1} a-\theta_{2} a<0$ and $\lim _{x \rightarrow \rho+} \int_{x}^{1} \frac{\gamma}{y-\rho} \mathrm{d} y=+\infty$, one has $\lim _{x \rightarrow \rho+} \Phi(x)=-\infty$. Based on the above analysis, we conclude that $(4.32)$ has a unique root $q_{0}=q(0) \in(\rho, 1)$ if and only if the inequality

$$
P<\frac{k_{3} a}{\delta}\left(\theta_{1}-\frac{1}{2} \theta_{2}\right)
$$

is valid. Consequently, the values of $x_{0}=Q(1)$ and $u_{0}$ in (4.25) are also obtained.

Next, we define

$$
I(\xi):=\int_{0}^{\xi}\left(f^{\prime}(x)-\beta_{2}\right) \mathrm{d} x=f(\xi)-f(0)-\beta_{2} \xi,
$$

where $f(x)$ is of the form (4.29). Note that $I(\xi)$ is decreasing with respect to $\beta_{2}$. We confirm that $f(x)$ in $(4.29)$ satisfies (4.5), according to the following cases.

(1) In the case of $\beta_{1}<f^{\prime}(0)=k_{3} e^{\int_{0}^{x_{0}} \frac{\theta_{2} a}{b^{2} q(z)} \mathrm{d} z} \leq \beta_{2}$, we have $f^{\prime}(x) \leq \beta_{2}$ for all $x \geq 0$, as $f^{\prime}(x)$ is decreasing on $[0, \infty)$. Thus, $\mathscr{C} f(0)-f(0)=\max _{y \geq 0}\left\{f(y)-\beta_{2} y-K\right\}-f(0)=-K<0,(4.5)$ is established. Figure $1(\mathrm{a})$ is a graph of $f^{\prime}(x)$ in this case.

(2) In the case of $f^{\prime}(0)=k_{3} e^{\int_{0}^{x_{0}} \frac{\theta_{2} a}{b^{2} q(z)} \mathrm{d} z}>\beta_{2} \geq k_{3}, f^{\prime}(x)$ is strictly decreasing from $f^{\prime}(0)$ to $f^{\prime}\left(u_{0}\right)=\beta_{1}$ and $f^{\prime}\left(x_{0}\right)=k_{3}$. Then, there exists a unique number $\xi_{1}^{*} \in\left(0, x_{0}\right)$, such that $f^{\prime}\left(\xi_{1}^{*}\right)=\beta_{2}$. (4.5) holds if and only if

$$
K \geq I\left(\xi_{1}^{*}\right),
$$

where $I(\xi)$ is defined in (4.34). Figure $1(\mathrm{~b})$ provides a graph of $f^{\prime}(x)$ in this case.

(3) In the case of $f^{\prime}(0)=k_{3} e^{\int_{0}^{x_{0}} \frac{\theta_{2} a}{b^{2} q(z)} \mathrm{d} z}>k_{3} \geq \beta_{2}$, for the same reason as above, there exists a unique number $\xi_{2}^{*} \in\left(x_{0}, u_{0}\right)$, such that $f^{\prime}\left(\xi_{2}^{*}\right)=\beta_{2}$. Define the integral

$$
J\left(\xi_{2}^{*}\right):=\int_{x_{0}}^{\xi_{2}^{*}}\left(f^{\prime}(x)-\beta_{2}\right) \mathrm{d} x=f\left(\xi_{2}^{*}\right)-f\left(x_{0}\right)-\beta_{2}\left(\xi_{2}^{*}-x_{0}\right),
$$

where $f(x)$ is of the form (4.29). $J\left(\xi_{2}^{*}\right)$ is also decreasing with respect to $\beta_{2}$. (4.5) holds if and only if

$$
K \geq I\left(\xi_{2}^{*}\right) .
$$

Again, where $I(\xi)$ is defined in (4.34). Figure 1(c) is a graph of $f^{\prime}(x)$ in this case. Eq. (4.36) is used later.

Considering the opposite of (4.33), for

$$
P \geq \frac{k_{3} a}{\delta}\left(\theta_{1}-\frac{1}{2} \theta_{2}\right)
$$




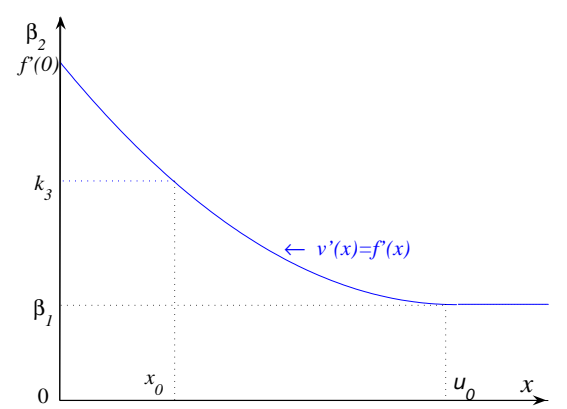

(a)

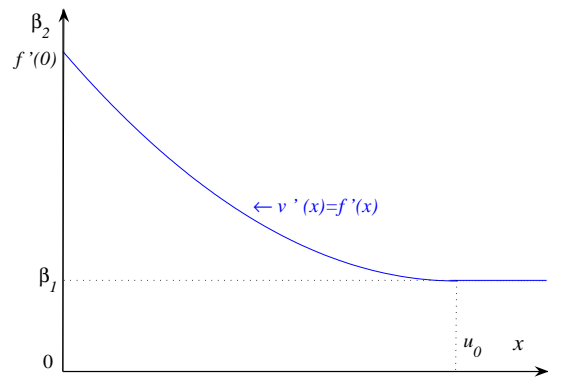

(d)

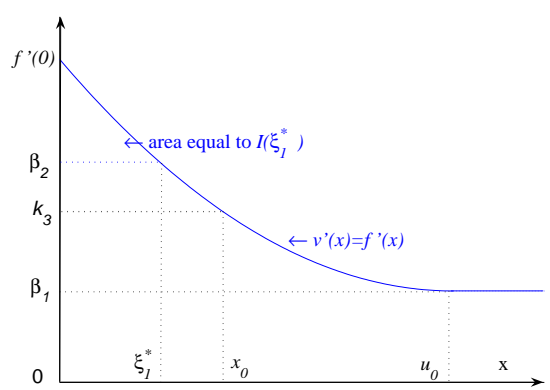

(b)

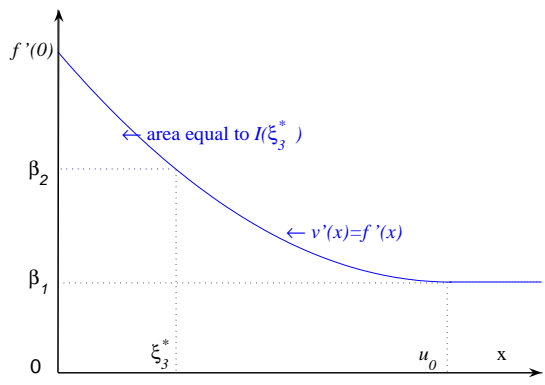

(e)

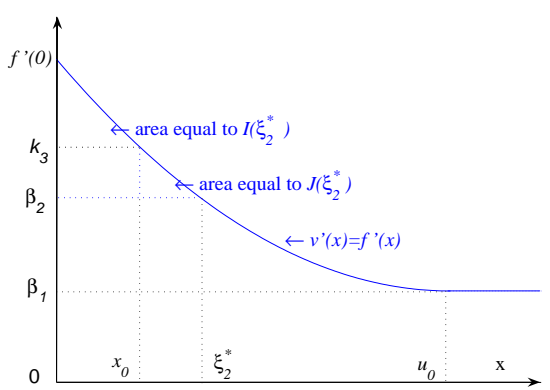

(c)

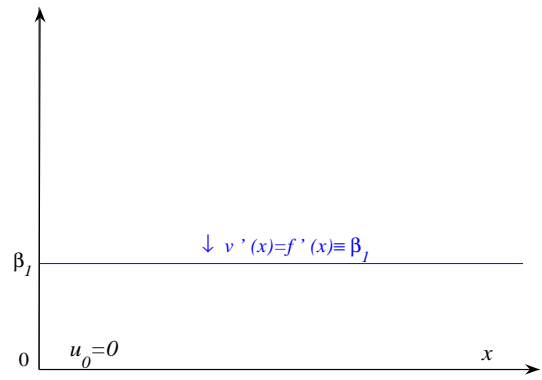

(f)

Figure 1: The graph of the derivative $v^{\prime}(x)=f^{\prime}(x)$ without financing

(a) $P<\frac{k_{3} a}{\delta}\left(\theta_{1}-\frac{1}{2} \theta_{2}\right), \beta_{1}<f^{\prime}(0)=k_{3} e^{\int_{0}^{x_{0}} \frac{\theta_{2} a}{b^{2} q(z)} \mathrm{d} z} \leq \beta_{2}$;

(b) $P<\frac{k_{3} a}{\delta}\left(\theta_{1}-\frac{1}{2} \theta_{2}\right), f^{\prime}(0)=k_{3} e^{\int_{0}^{x_{0}} \frac{\theta_{2} a}{b^{2} q(z)} \mathrm{d} z}>\beta_{2} \geq k_{3}, K \geq I\left(\xi_{1}^{*}\right)$;

(c) $P<\frac{k_{3} a}{\delta}\left(\theta_{1}-\frac{1}{2} \theta_{2}\right), f^{\prime}(0)=k_{3} e^{\int_{0}^{x_{0}} \frac{\theta_{2} a}{b^{2} q(z)} \mathrm{d} z}>k_{3} \geq \beta_{2}, K \geq I\left(\xi_{2}^{*}\right)$;

(d) $\frac{k_{3} a}{\delta}\left(\theta_{1}-\frac{1}{2} \theta_{2}\right) \leq P<\frac{\beta_{1} \theta_{1} a}{\delta}, f^{\prime}(0)=k_{1} r_{+}+k_{2} r_{-} \leq \beta_{2}$;

(e) $\frac{k_{3} a}{\delta}\left(\theta_{1}-\frac{1}{2} \theta_{2}\right) \leq P<\frac{\beta_{1} \theta_{1} a}{\delta}, f^{\prime}(0)=k_{1} r_{+}+k_{2} r_{-}>\beta_{2}, K \geq I\left(\xi_{3}^{*}\right)$;

(f) $P \geq \frac{\beta_{1} \theta_{1} a}{\delta}$.

(4.32) has no solution on $(\rho, 1)$. We set $q(x) \equiv 1$, i.e., the insurer does not use reinsurance at all. An intuitive interpretation is that the insurer is willing to take all risks if the "salvage value" is large enough. A suggested solution to (4.2)-(4.4) is of the form

$$
f(x)= \begin{cases}\beta_{1}\left(x-u_{0}\right)+f\left(u_{0}\right), & x \geq u_{0}, \\ k_{1} e^{r_{+} x}+k_{2} e^{r_{-} x}, & 0 \leq x \leq u_{0},\end{cases}
$$

with some $u_{0}>0$. The principle of smooth fit at $u_{0}$ results in

$$
\begin{aligned}
& k_{1}=\frac{\beta_{1} r_{-}}{r_{+}\left(r_{-}-r_{+}\right)} e^{-r_{+} u_{0}}>0, \\
& k_{2}=\frac{\beta_{1} r_{+}}{r_{-}\left(r_{+}-r_{-}\right)} e^{-r_{-} u_{0}}<0 .
\end{aligned}
$$

The switching level $u_{0}$ can be determined by boundary condition (4.4); specifically,

$$
\frac{\beta_{1} r_{-}}{r_{+}\left(r_{-}-r_{+}\right)} e^{-r_{+} u_{0}}+\frac{\beta_{1} r_{+}}{r_{-}\left(r_{+}-r_{-}\right)} e^{-r_{-} u_{0}}=P \text {. }
$$

To prove the existence of $u_{0}$, we consider the following function

$$
w(x):=\frac{\beta_{1} r_{-}}{r_{+}\left(r_{-}-r_{+}\right)} e^{-r_{+} x}+\frac{\beta_{1} r_{+}}{r_{-}\left(r_{+}-r_{-}\right)} e^{-r_{-} x},
$$

for $x \geq 0$. It is easy to see that $w(0)=\frac{\beta_{1}\left(r_{+}+r_{-}\right)}{r_{+} r_{-}}=\frac{\beta_{1} \theta_{1} a}{\delta}>0, w^{\prime}(x)<0$ and $w(\infty)=-\infty$, which imply that $w(x)$ is strictly decreasing from $\frac{\beta_{1} \theta_{1} a}{\delta}$ to $-\infty$. Thus, under the condition of

$$
\frac{k_{3} a}{\delta}\left(\theta_{1}-\frac{1}{2} \theta_{2}\right) \leq P<\frac{\beta_{1} \theta_{1} a}{\delta}
$$


(4.42) has a unique solution $u_{0} \in\left(0, \frac{1}{r_{+}-r_{-}} \ln \left(\frac{b^{2}+\theta_{2} a / r_{+}}{b^{2}+\theta_{2} a / r_{-}}\right)\right]$, which is a decreasing function of $P$. The above inequality suggests that

$$
\frac{k_{3} a}{\delta}\left(\theta_{1}-\frac{1}{2} \theta_{2}\right)<\frac{\beta_{1} \theta_{1} a}{\delta} .
$$

Recall the expression of $k_{3}$. We rewrite inequality (4.44) as

$$
\frac{b^{2}}{b^{2}+\frac{\theta_{2} a}{r_{+}}}\left(\frac{b^{2}+\frac{\theta_{2} a}{r_{+}}}{b^{2}+\frac{\theta_{2} a}{r_{-}}}\right)^{\frac{-r_{-}}{r_{+}-r_{-}}}\left(\theta_{1}-\frac{1}{2} \theta_{2}\right)<\theta_{1} .
$$

The proof of (4.45) can be found in Appendix B. Then, we check inequality (4.5) when $f(x)$ is of the form (4.39).

(1) In the case of $f^{\prime}(0)=k_{1} r_{+}+k_{2} r_{-}=\frac{\beta_{1} r_{-}}{r_{-}-r_{+}} e^{-r_{+} u_{0}}+\frac{\beta_{1} r_{+}}{r_{+}-r_{-}} e^{-r_{-} u_{0}} \leq \beta_{2}$, it has $f^{\prime}(x) \leq \beta_{2}$ for all $x \geq 0$, as $f^{\prime}(x)$ is decreasing on $[0, \infty)$. Hence, $\mathscr{C} f(0)-f(0)=\max _{y \geq 0}\left\{f(y)-\beta_{2} y-K\right\}-f(0)=-K<0$ and (4.5) follows. Figure 1(d) is a graph of $f^{\prime}(x)$ in this case.

(2) In the case of $f^{\prime}(0)=k_{1} r_{+}+k_{2} r_{-}=\frac{\beta_{1} r_{-}}{r_{-}-r_{+}} e^{-r_{+} u_{0}}+\frac{\beta_{1} r_{+}}{r_{+}-r_{-}} e^{-r_{-} u_{0}}>\beta_{2}, f^{\prime}(x)$ is strictly decreasing from $f^{\prime}(0)$ to $f^{\prime}\left(u_{0}\right)=\beta_{1}$. Then, there exists a unique number $\xi_{3}^{*} \in\left(0, u_{0}\right)$, such that $f^{\prime}\left(\xi_{3}^{*}\right)=\beta_{2}$. Apparently, (4.5) holds if and only if

$$
K \geq I\left(\xi_{3}^{*}\right),
$$

where $I(\xi)$ is defined by (4.34) with $f(x)$ in (4.39). Figure $1(\mathrm{e})$ is a graph of $f^{\prime}(x)$ in this case.

Finally, we are in the position to consider the last case

$$
P \geq \frac{\beta_{1} \theta_{1} a}{\delta} .
$$

Then, (4.42) has no positive solution. In this case, we set $u_{0}=0$, which means that the insurer pays all of the current surplus as dividends and claims the liquidation value immediately. The corresponding performance function is

$$
f(x)=\beta_{1} x+P, \quad x \geq 0,
$$

which is indeed a solution to (4.2)-(4.5), and the proof is omitted. Figure 1(f) is a graph of $f^{\prime}(x)$ in this case.

Remark 4.1. Consider the special case $P=0$, as in Taksar (2000a). If $\theta_{1}<\theta_{2} \leq 2 \theta_{1}$, then (4.32) has a unique solution $q_{0}=2\left(\theta_{2}-\theta_{1}\right) / \theta_{2} \in(\rho, 1]$ and the value function takes the form of (4.29). However, for $2 \theta_{1}<\theta_{2}$, there is no solution to (4.32) on the interval $(\rho, 1]$ because $2\left(\theta_{2}-\theta_{1}\right) / \theta_{2}>1$. The value function coincides with $f(x)$ in (4.39) because condition (4.43) holds. These results are consistent with those in Theorem 6.1 of Taksar (2000a).

\subsection{The case without bankruptcy}

The analysis above proves that no appropriate solution satisfies (4.2)-(4.5) simultaneously when (4.35), (4.37) or (4.46) fails. In other words, it is no longer optimal to withdraw from the market when the surplus is null. We now consider the second case with $v(0) \geq P$ and $\mathscr{C} v(0)-v(0)=0$; that is, the manager should raise an appropriate amount of capital to prevent bankruptcy. Then, the solution $f(x)$ for $v(x)$ should satisfy

$$
\begin{aligned}
& \max _{0 \leq q \leq 1}\left\{\mathscr{A}^{q} f(x)\right\}=0,0<x \leq \tilde{u}_{0}, \\
& \beta_{1}-f^{\prime}(x)=0, \quad x \geq \tilde{u}_{0}, \\
& f(0) \geq P \\
& \mathscr{C} f(0)-f(0)=0
\end{aligned}
$$

with some parameter $\tilde{u}_{0} \geq 0$. In fact, (4.49)-(4.52) establish that the continuation region is $\left(0, \tilde{u}_{0}\right]$, the dividend region is $\left(\tilde{u}_{0}, \infty\right)$ and the financing region is $\{0\}$. In what follows, we solve (4.49)-(4.52) when (4.35), (4.37) or (4.46) fails. There are four cases to be discussed. 
(1) If $P<\frac{k_{3} a}{\delta}\left(\theta_{1}-\frac{1}{2} \theta_{2}\right), f^{\prime}(0)=k_{3} e^{\int_{0}^{x_{0}} \frac{\theta_{2} a}{b^{2} q(z)} \mathrm{d} z}>\beta_{2} \geq k_{3}$ and $K<I\left(\xi_{1}^{*}\right)$ hold, let $x_{0}=Q(1)$ and $u_{0}, f(x)$ and $q^{\pi^{*}}(x)$ be given in $(4.25),(4.29)$ and (4.30), respectively. We give a candidate solution $f_{1}(x)=f\left(x+p_{1}^{*}\right)$ with some parameter $p_{1}^{*}>0$, i.e.,

$$
f_{1}(x)= \begin{cases}\beta_{1}\left(x-u_{1}\right)+f_{1}\left(u_{1}\right), & x \geq u_{1}, \\ k_{1} e^{r_{+}\left(x-x_{1}\right)}+k_{2} e^{r-\left(x-x_{1}\right)}, & x_{1} \leq x \leq u_{1}, \\ k_{3} \int_{0}^{x+p_{1}^{*}} e^{\int_{y}^{x_{0}} \frac{\theta_{2} a}{b^{2} q(z)} \mathrm{d} z} \mathrm{~d} y+P, & 0 \leq x \leq x_{1},\end{cases}
$$

where $x_{1}=x_{0}-p_{1}^{*}>0$ and $u_{1}=u_{0}-p_{1}^{*}>0$. Given (4.6), we define the candidate optimal reinsurance policy as $q_{1}^{\pi^{*}}(x)=q^{\pi^{*}}\left(x+p_{1}^{*}\right)$, i.e.,

$$
q_{1}^{\pi^{*}}(x)= \begin{cases}1, & x \geq x_{1}, \\ Q^{-1}\left(x+p_{1}^{*}\right), & 0 \leq x \leq x_{1} .\end{cases}
$$

It is easy to see that $f_{1}(x)$ and $q_{1}^{\pi^{*}}(x)$ satisfy (4.49)-(4.51) with $\tilde{u}_{0}=u_{1}$, as they can be obtained by shifting $f(x)$ and $q^{\pi^{*}}(x)$ to the left $p_{1}^{*}$ units, respectively. A graph of $v^{\prime}(x)=f_{1}^{\prime}(x)$ can be found in Figure 2(a). Now we need to determine the value of $p_{1}^{*}>0$, such that (4.52) holds. Define a function of $p, \varphi(p)=\varphi(p ; \xi)$, as

$$
\varphi(p)=\varphi(p ; \xi):=f(\xi)-f(p)-\beta_{2}(\xi-p)-K, \quad 0 \leq p \leq \xi .
$$

The condition $K<I\left(\xi_{1}^{*}\right)$ leads to

$$
\varphi\left(0 ; \xi_{1}^{*}\right)=f\left(\xi_{1}^{*}\right)-f(0)-\beta_{2} \xi_{1}^{*}-K=I\left(\xi_{1}^{*}\right)-K>0 .
$$

In addition, we have

$$
\begin{aligned}
& \varphi\left(\xi_{1}^{*} ; \xi_{1}^{*}\right)=-K<0, \\
& \varphi^{\prime}\left(p ; \xi_{1}^{*}\right)=\beta_{2}-f^{\prime}(p)<0 .
\end{aligned}
$$

Thus, there exists a unique solution $p_{1}^{*} \in\left(0, \xi_{1}^{*}\right)$ that satisfies $\varphi\left(p_{1}^{*} ; \xi_{1}^{*}\right)=0$ or, equivalently,

$$
f_{1}\left(\eta_{1}^{*}\right)-f_{1}(0)-\beta_{2} \eta_{1}^{*}-K=0,
$$

where $\eta_{1}^{*}:=\xi_{1}^{*}-p_{1}^{*}>0$. Noting that $f_{1}^{\prime}\left(\eta_{1}^{*}\right)=f^{\prime}\left(\xi_{1}^{*}\right)=\beta_{2}$, then (4.52) comes from

$$
\mathscr{C} f_{1}(0)=\max _{y \geq 0}\left\{f_{1}(y)-\beta_{2} y-K\right\}=f_{1}\left(\eta_{1}^{*}\right)-\beta_{2} \eta_{1}^{*}-K=f_{1}(0) .
$$

Similarly, we can present the solutions to (4.49)-(4.52) in other cases, but we omit the details here.

(2) If $P<\frac{k_{3} a}{\delta}\left(\theta_{1}-\frac{1}{2} \theta_{2}\right), f^{\prime}(0)=k_{3} e^{\int_{0}^{x_{0}} \frac{\theta_{2} a}{b^{2} q(z)} \mathrm{d} z}>k_{3} \geq \beta_{2}$ and $J\left(\xi_{2}^{*}\right)<K<I\left(\xi_{2}^{*}\right)$ hold, let $u_{0}, f(x)$ and $q^{\pi^{*}}(x)$ be given by $(4.25),(4.29)$ and $(4.30)$, respectively. Define a candidate solution $f_{2}(x)=f\left(x+p_{2}^{*}\right)$

$$
f_{2}(x)= \begin{cases}\beta_{1}\left(x-u_{2}\right)+f_{2}\left(u_{2}\right), & x \geq u_{2}, \\ k_{1} e^{r_{+}\left(x-x_{2}\right)}+k_{2} e^{r_{-}\left(x-x_{2}\right)}, & x_{2} \leq x \leq u_{2}, \\ k_{3} \int_{0}^{x+p_{2}^{*}} e^{\int_{y}^{x_{0}} \frac{\theta_{2} a}{b^{2} q(z)} \mathrm{d} z} \mathrm{~d} y+P, & 0 \leq x \leq x_{2},\end{cases}
$$

where $x_{2}=x_{0}-p_{2}^{*}>0, u_{2}=u_{0}-p_{2}^{*}>0$ and $p_{2}^{*} \in\left(0, x_{0}\right)$ is the unique solution to $\varphi\left(p ; \xi_{2}^{*}\right)=0$ or, equivalently,

$$
f_{2}\left(\eta_{2}^{*}\right)-f_{2}(0)-\beta_{2} \eta_{2}^{*}-K=0,
$$

where $\eta_{2}^{*}:=\xi_{2}^{*}-p_{2}^{*}>0$. Accordingly, define a candidate optimal reinsurance policy $q_{2}^{\pi^{*}}(x)=q^{\pi^{*}}\left(x+p_{2}^{*}\right)$

$$
q_{2}^{\pi^{*}}(x)= \begin{cases}1, & x \geq x_{2} \\ Q^{-1}\left(x+p_{2}^{*}\right), & 0 \leq x<x_{2}\end{cases}
$$

Then, $f_{2}(x)$ and $q_{2}^{\pi^{*}}(x)$ satisfy (4.49)-(4.52) with $\tilde{u}_{0}=u_{2}$. Figure 2(b) is a graph of $v^{\prime}(x)=f_{2}^{\prime}(x)$ in this case. 
(3) If $P<\frac{k_{3} a}{\delta}\left(\theta_{1}-\frac{1}{2} \theta_{2}\right), f^{\prime}(0)=k_{3} e^{\int_{0}^{x_{0}} \frac{\theta_{2} a}{b^{2} q(z)} \mathrm{d} z}>k_{3} \geq \beta_{2}$ and $0<K \leq J\left(\xi_{2}^{*}\right)<I\left(\xi_{2}^{*}\right)$ hold, let $u_{0}, f(x)$ and $q^{\pi^{*}}(x)$ be given by (4.25), (4.29) and (4.30), respectively. Define a candidate solution $f_{3}(x)=f\left(x+p_{3}^{*}\right)$

$$
f_{3}(x)= \begin{cases}\beta_{1}\left(x-u_{3}\right)+f_{3}\left(u_{3}\right), & x \geq u_{3}, \\ k_{1} e^{r_{+}\left(x-u_{3}\right)}+k_{2} e^{r_{-}\left(x-u_{3}\right)}, & 0 \leq x \leq u_{3},\end{cases}
$$

where $u_{3}=u_{0}-p_{3}^{*}>0$ and $p_{3}^{*} \in\left[x_{0}, \xi_{2}^{*}\right)$ is the unique solution to $\varphi\left(p ; \xi_{2}^{*}\right)=0$ or, equivalently,

$$
f_{3}\left(\eta_{3}^{*}\right)-f_{3}(0)-\beta_{2} \eta_{3}^{*}-K=0
$$

where $\eta_{3}^{*}:=\xi_{2}^{*}-p_{3}^{*}>0$. Correspondingly, define a reinsurance policy

$$
q_{3}^{\pi^{*}}(x)=q^{\pi^{*}}\left(x+p_{3}^{*}\right) \equiv 1, x \geq 0 .
$$

Then, $f_{3}(x)$ and $q_{3}^{\pi^{*}}(x)$ satisfy (4.49)-(4.52) with $\tilde{u}_{0}=u_{3}$. Figure $2(\mathrm{c})$ is a graph of $v^{\prime}(x)=f_{3}^{\prime}(x)$ in this case.

(4) If $\frac{k_{3} a}{\delta}\left(\theta_{1}-\frac{1}{2} \theta_{2}\right) \leq P<\frac{\beta_{1} \theta_{1} a}{\delta}, f^{\prime}(0)=k_{1} r_{+}+k_{2} r_{-}>\beta_{2}$ and $K<I\left(\xi_{3}^{*}\right)$ hold, let $f(x)$ and $u_{0}$ be defined by (4.39) and (4.42), respectively. Define a candidate solution $f_{4}(x)=f\left(x+p_{4}^{*}\right)$

$$
f_{4}(x)= \begin{cases}\beta_{1}\left(x-u_{4}\right)+f_{4}\left(u_{4}\right), & x \geq u_{4} \\ k_{1} e^{r_{+}\left(x-u_{4}\right)}+k_{2} e^{r_{-}\left(x-u_{4}\right)}, & 0 \leq x \leq u_{4}\end{cases}
$$

where $u_{4}=u_{0}-p_{4}^{*}$ and $p_{4}^{*} \in\left(0, \xi_{3}^{*}\right)$ is the unique solution of $\varphi\left(p ; \xi_{3}^{*}\right)=0$ or, equivalently,

$$
f_{4}\left(\eta_{4}^{*}\right)-f_{4}(0)-\beta_{2} \eta_{4}^{*}-K=0
$$

where $\eta_{4}^{*}:=\xi_{3}^{*}-p_{4}^{*}$. Define a reinsurance policy by

$$
q_{4}^{\pi^{*}}(x) \equiv 1, \quad x \geq 0 .
$$

Then, $f_{4}(x)$ and $q_{4}^{\pi^{*}}(x)$ solve (4.49)-(4.52) with $\tilde{u}_{0}=u_{4}$. Figure $2(\mathrm{~d})$ is a graph of $f_{4}^{\prime}(x)$ in this case.

\subsection{The value function and optimal strategy}

Based on the analysis above, we identify the explicit solution to the value function and construct the associated optimal strategy in this section. Before presenting the main results, we introduce a definition

$$
D_{t}^{\pi^{*}}(u)=(x-u)_{+}+\int_{0}^{t} I_{\left\{X_{s}^{\pi^{*}} \geq u\right\}} \mathrm{d} D_{s}^{\pi^{*}}
$$

Theorem 4.1. Under the assumption of (4.1), $V(x)$ and $\pi^{*}$ can be obtained in the following 10 cases, which exhaust all of the possibilities. In what follows, $k_{3}, I(\xi)$ and $J\left(\xi_{2}^{*}\right)$ are defined as $(4.26),(4.34)$ and (4.36), respectively.

Case 1: $P<\frac{k_{3} a}{\delta}\left(\theta_{1}-\frac{1}{2} \theta_{2}\right)$ and $\beta_{1}<f^{\prime}(0)=k_{3} e^{\int_{0}^{x_{0}} \frac{\theta_{2} a}{b^{2} q(z)} \mathrm{d} z} \leq \beta_{2}$

In this case, let $u_{0}$ and $f(x)$ be given by (4.25) and (4.29), respectively. The value function $V(x)$ coincides with $f(x)$. The surplus process controlled by the optimal strategy $\pi^{*}=\left(q^{\pi^{*}}, D^{\pi^{*}}, R^{\pi^{*}}\right)$ satisfies that

$$
\left\{\begin{array}{l}
X_{t}^{\pi^{*}}=x+\int_{0}^{t}\left(a\left(\theta_{1}-\left(1-q^{\pi^{*}}\left(X_{s}^{\pi^{*}}\right)\right) \theta_{2}\right)\right) \mathrm{d} s+\int_{0}^{t} q^{\pi^{*}}\left(X_{s}^{\pi^{*}}\right) b \mathrm{~d} B_{s}-D_{t}^{\pi^{*}} \\
X_{t}^{\pi^{*}} \leq u_{0} .
\end{array}\right.
$$

The optimal reinsurance policy $q^{\pi^{*}}$ is determined by (4.30). $D^{\pi^{*}}$ is a barrier dividend strategy with switch level $u_{0}$, which is described by (4.67), where $u_{i}=u_{0}>0$. In this case, it is unprofitable to raise new money, so $R_{t}^{\pi^{*}} \equiv 0$.

Case 2: $P<\frac{k_{3} a}{\delta}\left(\theta_{1}-\frac{1}{2} \theta_{2}\right), f^{\prime}(0)=k_{3} e^{\int_{0}^{x_{0}} \frac{\theta_{2} a}{b^{2} q(z)} \mathrm{d} z}>\beta_{2} \geq k_{3}$ and $K \geq I\left(\xi_{1}^{*}\right)$

The value function $V(x)$ and associated optimal strategy $\pi^{*}$ take the same forms as those in Case 1 .

Case 3: $P<\frac{k_{3} a}{\delta}\left(\theta_{1}-\frac{1}{2} \theta_{2}\right), f^{\prime}(0)=k_{3} e^{\int_{0}^{x_{0}} \frac{\theta_{2} a}{b^{2} q(z)} \mathrm{d} z}>k_{3} \geq \beta_{2}$ and $K \geq I\left(\xi_{2}^{*}\right)$ 


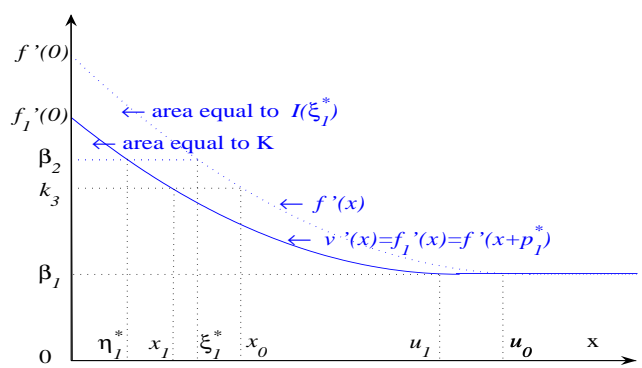

(a)

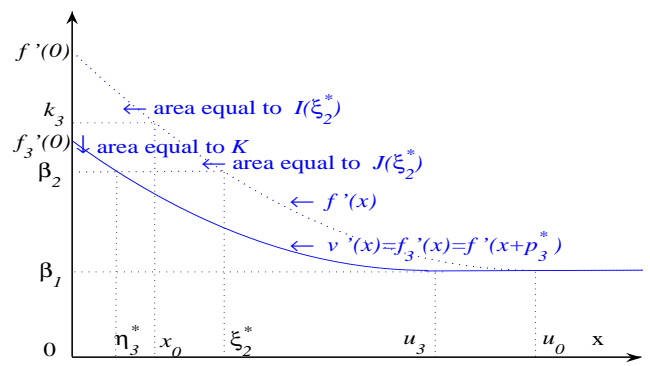

(c)

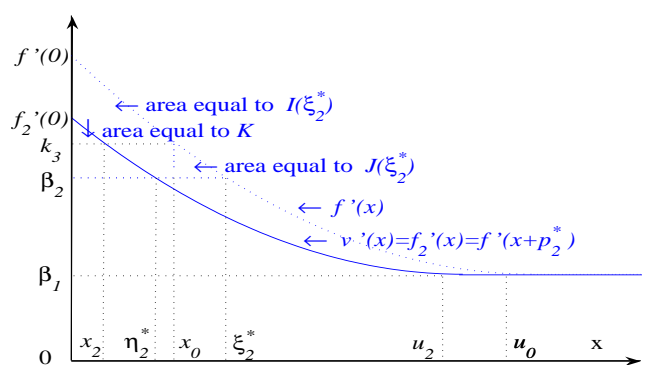

(b)

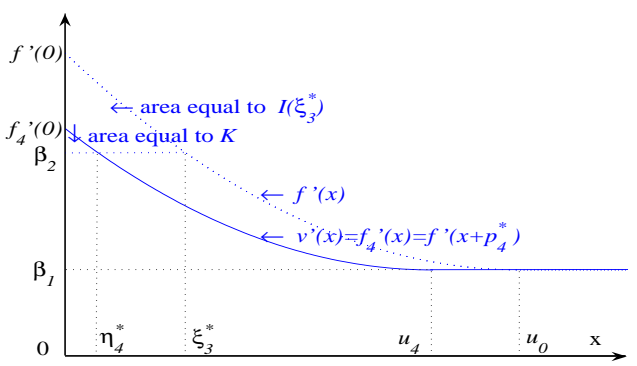

(d)

Figure 2: The graph of the derivative $v^{\prime}(x)=f_{i}^{\prime}(x)$ with forced financing
(a) $P<\frac{k_{3} a}{\delta}\left(\theta_{1}-\frac{1}{2} \theta_{2}\right), f^{\prime}(0)=k_{3} e^{\int_{0}^{x_{0}} \frac{\theta_{2} a}{b^{2} q(z)} \mathrm{d} z}>\beta_{2} \geq k_{3}$ and $0<K<I\left(\xi_{1}^{*}\right)$;
(b) $P<\frac{k_{3} a}{\delta}\left(\theta_{1}-\frac{1}{2} \theta_{2}\right), f^{\prime}(0)=k_{3} e^{\int_{0}^{x_{0}} \frac{\theta_{2} a}{b^{2} q(z)} \mathrm{d} z}>k_{3} \geq \beta_{2}$ and $J\left(\xi_{2}^{*}\right)<K<I\left(\xi_{2}^{*}\right)$;
(c) $P<\frac{k_{3} a}{\delta}\left(\theta_{1}-\frac{1}{2} \theta_{2}\right), f^{\prime}(0)=k_{3} e^{\int_{0}^{x_{0}} \frac{\theta_{2} a}{b^{2} q(z)} \mathrm{d} z}>k_{3} \geq \beta_{2}$ and $0<K \leq J\left(\xi_{2}^{*}\right)<I\left(\xi_{2}^{*}\right)$;
(d) $\frac{k_{3} a}{\delta}\left(\theta_{1}-\frac{1}{2} \theta_{2}\right) \leq P<\frac{\beta_{1} \theta_{1} a}{\delta}, f^{\prime}(0)=k_{1} r_{+}+k_{2} r_{-}>\beta_{2}$ and $0<K<I\left(\xi_{3}^{*}\right)$.

The value function $V(x)$ and associated optimal strategy $\pi^{*}$ take the same forms as those in Case 1.

Case 4: $\frac{k_{3} a}{\delta}\left(\theta_{1}-\frac{1}{2} \theta_{2}\right) \leq P<\frac{\beta_{1} \theta_{1} a}{\delta}$ and $f^{\prime}(0)=k_{1} r_{+}+k_{2} r_{-} \leq \beta_{2}$

In this case, let $f(x)$ and $u_{0}$ be given by (4.39) and (4.42), respectively. The value function $V(x)$ is consistent with $f(x)$. The controlled surplus process associated with optimal strategy $\pi^{*}=\left(q^{\pi^{*}}, D^{\pi^{*}}, R^{\pi^{*}}\right)$ satisfies that

$$
\left\{\begin{array}{l}
X_{t}^{\pi^{*}}=x+\theta_{1} a t+b B_{t}-D_{t}^{\pi^{*}} \\
X_{t}^{\pi^{*}} \leq u_{0}
\end{array}\right.
$$

The insurer should take full retention all the time; that is, $q_{t}^{\pi^{*}} \equiv 1$, the barrier dividend strategy $D^{\pi^{*}}$ with switch level $u_{0}>0$ is optimal, which is described by (4.67), where $u=u_{0}$. It is unprofitable to raise new money all of the time, so $R_{t}^{\pi^{*}} \equiv 0$.

Case 5: $\frac{k_{3} a}{\delta}\left(\theta_{1}-\frac{1}{2} \theta_{2}\right) \leq P<\frac{\beta_{1} \theta_{1} a}{\delta}, f^{\prime}(0)=k_{1} r_{+}+k_{2} r_{-}>\beta_{2}$ and $K \geq I\left(\xi_{3}^{*}\right)$

The value function $V(x)$ and $\pi^{*}$ take the same forms as those in Case 4.

Case 6: $P \geq \frac{\beta_{1} \theta_{1} a}{\delta}$

The value function $V(x)$ coincides with $f(x)$ in (4.48). It is optimal to distribute all of the surplus $x$ as dividends and immediately claim the liquidation value at bankruptcy time. Mathematically, $q^{\pi^{*}} \equiv 0, D_{t}^{\pi^{*}} \equiv x$ and $R_{t}^{\pi^{*}} \equiv 0$.

Case 7: $P<\frac{k_{3} a}{\delta}\left(\theta_{1}-\frac{1}{2} \theta_{2}\right), f^{\prime}(0)=k_{3} e^{\int_{0}^{x_{0}} \frac{\theta_{2} a}{b^{2} q(z)} \mathrm{d} z}>\beta_{2} \geq k_{3}$ and $K<I\left(\xi_{1}^{*}\right)$

Let $u_{0}$ be given by $(4.25)$ and $u_{1}=u_{0}-p_{1}^{*}$ in this case. Then, the value function $V(x)$ is identical to $f_{1}(x)$ in (4.53). The surplus controlled by the optimal strategy $\pi^{*}=\left(q_{1}^{\pi^{*}}, D^{\pi^{*}}, R^{\pi^{*}}\right)$ satisfies that

$$
\left\{\begin{array}{l}
X_{t}^{\pi^{*}}=x+\int_{0}^{t}\left(a\left(\theta_{1}-\left(1-q_{1}^{\pi^{*}}\left(X_{s}^{\pi^{*}}\right)\right) \theta_{2}\right)\right) \mathrm{d} s+\int_{0}^{t} q_{1}^{\pi^{*}}\left(X_{s}^{\pi^{*}}\right) b \mathrm{~d} B_{s}-D_{t}^{\pi^{*}}+\sum_{n=1}^{\infty} I_{\left\{\tau_{n}^{\pi^{*}} \leq t\right\}} \eta_{n}^{\pi^{*}} \\
0 \leq X_{t}^{\pi^{*}} \leq u_{1}
\end{array}\right.
$$


The optimal reinsurance policy $q_{1}^{\pi^{*}}$ is characterised by (4.54). The barrier dividend strategy $D^{\pi^{*}}$ with level $u_{1}$ is optimal, which is described by (4.67), where $u=u_{1}$. It is profitable to raise new money when and only when the surplus is null, and the surplus immediately jumps to $\eta_{1}^{*}=\xi_{1}^{*}-p_{1}^{*}$ once it reaches 0 by issuing equities. Thus, $R^{\pi^{*}}$ is characterised by

$$
\left\{\begin{array}{l}
\int_{0}^{\infty} I_{\left\{t: X_{t}^{\pi^{*}}>0\right\}} \mathrm{d} R_{t}^{\pi^{*}}=0 \\
\tau_{1}^{\pi^{*}}=\inf \left\{t \geq 0: X_{t-}^{\pi^{*}}=0\right\} \\
\tau_{n}^{\pi^{*}}=\inf \left\{t>\tau_{n-1}^{\pi^{*}}: X_{t-}^{\pi^{*}}=0\right\}, n=2,3, \cdots
\end{array}\right.
$$

and

$$
\eta_{n}^{\pi^{*}} \equiv \eta_{1}^{*}=\xi_{1}^{*}-p_{1}^{*}, n=1,2, \cdots
$$

Case 8: $P<\frac{k_{3} a}{\delta}\left(\theta_{1}-\frac{1}{2} \theta_{2}\right), f^{\prime}(0)=k_{3} e^{\int_{0}^{x_{0}} \frac{\theta_{2} a}{b^{2} q(z)} \mathrm{d} z}>k_{3} \geq \beta_{2}$ and $J\left(\xi_{2}^{*}\right)<K<I\left(\xi_{2}^{*}\right)$

Let $u_{0}$ be given by (4.25) and $u_{2}=u_{0}-p_{2}^{*}$ in this case. The value function $V(x)$ is identical to $f_{2}(x)$ in (4.58). The surplus process is controlled by the optimal strategy $\pi^{*}=\left(q_{2}^{\pi^{*}}, D^{\pi^{*}}, R^{\pi^{*}}\right)$ satisfies that

$$
\left\{\begin{array}{l}
X_{t}^{\pi^{*}}=x+\int_{0}^{t}\left(a\left(\theta_{1}-\left(1-q_{2}^{\pi^{*}}\left(X_{s}^{\pi^{*}}\right)\right) \theta_{2}\right)\right) \mathrm{d} s+\int_{0}^{t} q_{2}^{\pi^{*}}\left(X_{s}^{\pi^{*}}\right) b \mathrm{~d} B_{s}-D_{t}^{\pi^{*}}+\sum_{n=1}^{\infty} I_{\left\{\tau_{n}^{\pi^{*}} \leq t\right\}} \eta_{n}^{\pi^{*}} ; \\
0 \leq X_{t}^{\pi^{*}} \leq u_{2} .
\end{array}\right.
$$

The optimal reinsurance strategy $q_{2}^{\pi^{*}}$ is given by (4.60). The barrier dividend strategy $D^{\pi^{*}}$ with level $u_{2}$ is optimal, which is described by (4.67), where $u=u_{2}$. It is profitable to raise new money when and only when the surplus is zero, and the surplus immediately jumps to $\eta_{2}^{*}=\xi_{2}^{*}-p_{2}^{*}$ once it reaches 0 by issuing equities. Mathematically, $R^{\pi^{*}}$ can be characterised by (4.71) and

$$
\eta_{n}^{\pi^{*}} \equiv \eta_{2}^{*}=\xi_{2}^{*}-p_{2}^{*}, n=1,2, \cdots
$$

Case 9: $P<\frac{k_{3} a}{\delta}\left(\theta_{1}-\frac{1}{2} \theta_{2}\right), f^{\prime}(0)=k_{3} e^{\int_{0}^{x_{0}} \frac{\theta_{2} a}{b^{2} q(z)} \mathrm{d} z}>k_{3} \geq \beta_{2}$ and $0<K \leq J\left(\xi_{2}^{*}\right)<I\left(\xi_{2}^{*}\right)$

Let $u_{0}$ be given by $(4.25)$ and $u_{3}=u_{0}-p_{3}^{*}$ in this case. Then, $V(x)$ is identical to $f_{3}(x)$, as given by (4.61). The surplus process is controlled by the optimal strategy $\pi^{*}=\left(q_{3}^{\pi^{*}}, D^{\pi^{*}}, R^{\pi^{*}}\right)$ satisfies that

$$
\left\{\begin{array}{l}
X_{t}^{\pi^{*}}=x+\theta_{1} a t+b B_{t}-D_{t}^{\pi^{*}}+\sum_{n=1}^{\infty} I_{\left\{\tau_{n}^{\pi^{*}} \leq t\right\}} \eta_{n}^{\pi^{*}} \\
0 \leq X_{t}^{\pi^{*}} \leq u_{3}
\end{array}\right.
$$

The optimal reinsurance policy is $q_{3}^{\pi^{*}}(x) \equiv 1$. The barrier dividend strategy $D^{\pi^{*}}$ with switch level $u_{3}$ is optimal, which is described by (4.67), where $u=u_{3}$. It is profitable to raise new money when and only when the surplus is null, and the surplus immediately jumps to $\eta_{3}^{*}=\xi_{2}^{*}-p_{3}^{*}$ once it reaches 0 by issuing equities; that is, $R^{\pi^{*}}$ can be characterised by (4.71) and

$$
\eta_{n}^{\pi^{*}} \equiv \eta_{3}^{*}=\xi_{2}^{*}-p_{3}^{*}, n=1,2, \cdots .
$$

Case 10: $\frac{k_{3} a}{\delta}\left(\theta_{1}-\frac{1}{2} \theta_{2}\right) \leq P<\frac{\beta_{1} \theta_{1} a}{\delta}, f^{\prime}(0)=k_{1} r_{+}+k_{2} r_{-}>\beta_{2}$ and $0<K<I\left(\xi_{3}\right)$

Let $u_{0}$ be given by (4.42) and $u_{4}=u_{0}-p_{4}^{*}$ in this case. Then, $V(x)$ coincides with $f_{4}(x)$, as given by (4.64). The controlled surplus process associated with optimal strategy $\pi^{*}=\left(q_{4}^{\pi^{*}}, D^{\pi^{*}}, R^{\pi^{*}}\right)$ satisfies that

$$
\left\{\begin{array}{l}
X_{t}^{\pi^{*}}=x+\theta_{1} a t+b B_{t}-D_{t}^{\pi^{*}}+\sum_{n=1}^{\infty} I_{\left\{\tau_{n}^{\pi^{*}} \leq t\right\}} \eta_{n}^{\pi^{*}} \\
0 \leq X_{t}^{\pi^{*}} \leq u_{4}
\end{array}\right.
$$

The optimal reinsurance policy is $q_{4}^{\pi^{*}} \equiv 1$. The barrier dividend strategy $D^{\pi^{*}}$ with switch level $u_{4}$ is optimal, as described by(4.67), where $u=u_{4}$. It is profitable to raise new money when and only when the surplus is null, and the surplus immediately jumps to $\eta_{4}^{*}=\xi_{3}^{*}-p_{4}^{*}$ once it reaches 0 by issuing equities; that is, $R^{\pi^{*}}$ can be characterised by (4.71) and

$$
\eta_{n}^{\pi^{*}} \equiv \eta_{4}^{*}=\xi_{3}^{*}-p_{4}^{*}, n=1,2, \cdots
$$

Proof. We check that the function $V(x)$ given in the 10 cases above is increasing, concave and twice continuously differentiable, and that the derivative $V^{\prime}(x)$ is bounded. Substituting $V(x)$ in (3.1) and (3.2), we prove that $V(x)$ solves HJB equations. By applying Theorem 3.1, we establish that $V(x)$ and $\pi^{*}$ are solutions to Problem 2.1. We only provide the detailed proof of Case 7 in Appendix $\mathrm{C}$ as an example, even though the method is applicable to other cases. Hence, we have successfully obtained the solution to Problem 2.1. 
Remark 4.2. Observing Figures 1 and 2, we also draw several conclusions regarding the effects of the cost factors and liquidation value on optimal strategies.

(1) As Figure 1 shows, no financing is optimal when either the proportional factor $\beta_{2}$ or the fixed cost factor $K$ is too large, i.e., when $\beta_{2} \geq f^{\prime}(0)$ or $K \geq I\left(\xi_{i}^{*}\right)$. In Figures $1(\mathrm{a})$-(c), the dividend barrier $u_{0}$ and the initial ceded proportion $1-q^{\pi^{*}}(0)$ are increasing with respect to $\beta_{1}$ if $P \geq 0$. The opposite is true if $P<0$. In addition, both $u_{0}$ and $1-q^{\pi^{*}}(0)$ are decreasing in $P$, which means that the insurer would take higher risks with larger $P$ and would purchase no reinsurance once the liquidation value $P$ exceeded $\frac{k_{3} a}{\delta}\left(\theta_{1}-\frac{1}{2} \theta_{2}\right)$. See Figures 1(d)-(f). When the liquidation value $P$ is greater than $\frac{\beta_{1} \theta_{1} a}{\delta}$, it is optimal for the insurer to declare bankruptcy and claim the liquidation value immediately. Note that $\frac{\beta_{1} \theta_{1} a}{\delta}$ is the present value of a perpetuity with a discount rate of $\delta$ and an income rate of $\beta_{1} \theta_{1} a$, which is the expected after-tax profit rate under the full retention strategy. See risk model (2.5), where $q_{s}=1$, and Figure 1(f).

(2) As Figure 2 shows, the dividend barrier $u_{i}$, the amount of financing $\eta_{i}^{*}$ and the initial ceded proportion $1-q_{i}^{\pi^{*}}(0)$ are all increasing with respect to $K$. The interpretation is as follows. When the fixed cost $K$ increases, the manager should reserve more money and buy more reinsurance to protect against financial risk. $\mathrm{He} /$ she should also try to reduce the frequency of raising money from equity markets by enhancing the amount of $\eta_{i}^{*}$. The manager may consider financing only when both the conditions $\beta_{2}<f^{\prime}(0)$ and $K<I\left(\xi_{i}^{*}\right)$ hold. The insurer does not purchase any reinsurance once the liquidation value $P$ exceeds $\frac{k_{3} a}{\delta}\left(\theta_{1}-\frac{1}{2} \theta_{2}\right)$.

\section{The case of $\theta_{2} \in\left[\theta_{1}+\sqrt{\theta_{1}^{2}+2 \delta\left(\frac{b}{a}\right)^{2}}, \infty\right)$}

In this section, we address the case

$$
\theta_{2} \geq \theta_{1}+\sqrt{\theta_{1}^{2}+2 \delta\left(\frac{b}{a}\right)^{2}} .
$$

As in Section 4, we consider two sub-optimal problems, each corresponding to different boundary conditions. In particular, the proofs resemble those in Section 4, so we present the main results in this section but omit most of the proofs and interpretations.

\subsection{The case without financing}

As in the previous section, we start with the boundary conditions $v(0)=P$ and $\mathscr{C} v(0)-v(0) \leq 0$. In this case the solution $g(x)$ for $v(x)$ should satisfy that

$$
\begin{aligned}
& \max _{0 \leq q \leq 1}\left\{\mathscr{A}^{q} g(x)\right\}=0,0<x<d_{0}, \\
& \beta_{1}-g^{\prime}(x)=0, \quad x \geq d_{0}, \\
& g(0)=P \\
& \mathscr{C} g(0)-g(0) \leq 0
\end{aligned}
$$

with some parameter $d_{0} \geq 0$. Under condition (5.1), we conjecture that it is always optimal to buy no reinsurance; that is, $q(x) \equiv 1$ for all $x \geq 0$. Then, by solving (5.2) and (5.3) with $q \equiv 1$, we obtain an increasing, concave and twice continuously differentiable solution

$$
g(x)=\left\{\begin{array}{l}
\beta_{1}\left(x-d_{0}\right)+g\left(d_{0}\right), \quad x \geq d_{0}, \\
l_{1} e^{r_{+} x}+l_{2} e^{r_{-} x}, \quad 0 \leq x \leq d_{0} .
\end{array}\right.
$$

Using the principle of smooth fit at $d_{0}$, the constants $l_{1}$ and $l_{2}$ can be obtained by solving

$$
\begin{aligned}
& l_{1}=\frac{\beta_{1} r_{-}}{r_{+}\left(r_{-}-r_{+}\right)} e^{-r_{+} d_{0}}>0, \\
& l_{2}=\frac{\beta_{1} r_{+}}{r_{-}\left(r_{+}-r_{-}\right)} e^{-r_{-} d_{0}}<0 .
\end{aligned}
$$

The boundary condition (5.4) leads to the equation satisfied by $d_{0}$

$$
\frac{\beta_{1} r_{-}}{r_{+}\left(r_{-}-r_{+}\right)} e^{-r_{+} d_{0}}+\frac{\beta_{1} r_{+}}{r_{-}\left(r_{+}-r_{-}\right)} e^{-r_{-} d_{0}}=P .
$$


For the same reason as given for (4.42), there exists a unique solution $d_{0}>0$ to (5.9) if and only if $P<\frac{\beta_{1} \theta_{1} a}{\delta}$. Here, $d_{0}$ is also a decreasing function of $P$. To prove that $q(x) \equiv 1$ is optimal, given (4.6), it suffices to prove that

$$
G(x):=\frac{-\theta_{2} a g^{\prime}(x)}{b^{2} g^{\prime \prime}(x)}=\frac{\theta_{2} a}{b^{2}} \cdot \frac{-r_{-} e^{-r_{+}\left(d_{0}-x\right)}+r_{+} e^{-r_{-}\left(d_{0}-x\right)}}{r_{+} r_{-}\left(e^{-r_{+}\left(d_{0}-x\right)}-e^{-r_{-}\left(d_{0}-x\right)}\right)} \geq 1
$$

for all $x \in\left[0, d_{0}\right]$. Applying

$$
\theta_{2} \geq \theta_{1}+\sqrt{\theta_{1}^{2}+2 \delta\left(\frac{b}{a}\right)^{2}}=\frac{-b^{2}}{a} r_{-} \quad \text { and } \quad r_{+} r_{-}=\frac{-2 \delta}{b^{2}}
$$

we derive

$$
G(0)=\frac{\theta_{2} a}{b^{2}} \cdot \frac{-r_{-} e^{-r_{+} d_{0}}+r_{+} e^{-r_{-} d_{0}}}{r_{+} r_{-}\left(e^{-r_{+} d_{0}}-e^{-r_{-} d_{0}}\right)} \geq \frac{2 \delta e^{-r_{-} d_{0}}+\left(b r_{-}\right)^{2} e^{-r_{+} d_{0}}}{2 \delta e^{-r_{-} d_{0}}-2 \delta e^{-r_{+} d_{0}}}>1
$$

and

$$
G^{\prime}(x)=\frac{-\theta_{2} a\left(r_{+}-r_{-}\right)^{2}}{b^{2}\left(r_{+} r_{-}\right)^{3}\left(e^{-r_{+}\left(d_{0}-x\right)}-e^{-r_{-}\left(d_{0}-x\right)}\right)^{2}} \cdot e^{-\left(r_{+}+r_{-}\right)\left(d_{0}-x\right)}>0 .
$$

Thus, (5.10) is established by (5.11) and (5.12).

It is not difficult to see that $g^{\prime}(0)=\frac{\beta_{1}}{r_{+}-r_{-}}\left(r_{+} e^{-r_{-} d_{0}}-r_{-} e^{-r_{+} d_{0}}\right)>\beta_{1}$ for $d_{0}>0$. We check inequality (5.5) in two different cases.

(1) In the case where $\beta_{1}<g^{\prime}(0)=\frac{\beta_{1}}{r_{+}-r_{-}}\left(r_{+} e^{-r_{-} d_{0}}-r_{-} e^{-r_{+} d_{0}}\right) \leq \beta_{2}$, it has $g^{\prime}(x) \leq \beta_{2}$, as $g^{\prime}(x)$ is decreasing on $[0, \infty)$. Thus, $\mathscr{C} g(0)-g(0)=-K<0$, and (5.5) follows.

(2) In the case where $g^{\prime}(0)=\frac{\beta_{1}}{r_{+}-r_{-}}\left(r_{+} e^{-r_{-} d_{0}}-r_{-} e^{-r_{+} d_{0}}\right)>\beta_{2}$, there exists a unique number $\varsigma^{*} \in\left(0, d_{0}\right)$ such that $g^{\prime}\left(\varsigma^{*}\right)=\beta_{2}$, i.e.,

$$
\frac{\beta_{1} r_{-}}{r_{-}-r_{+}} e^{r_{+}\left(\varsigma^{*}-d_{0}\right)}+\frac{\beta_{-} r_{+}}{r_{+}-r_{-}} e^{r_{-}\left(\varsigma^{*}-d_{0}\right)}=\beta_{2}
$$

Define the integral

$$
H\left(\varsigma^{*}\right):=\int_{0}^{\varsigma^{*}}\left(g^{\prime}(x)-\beta_{2}\right) \mathrm{d} x=g\left(\varsigma^{*}\right)-g(0)-\beta_{2} \varsigma^{*},
$$

where $g(x)$ is defined by (5.6) and is decreasing with respect to $\beta_{2}$. Then, (5.5) holds if and only if

$$
K \geq H\left(\varsigma^{*}\right) .
$$

The opposite case is treated in the next subsection.

If $P \geq \frac{\beta_{1} \theta_{1} a}{\delta}$, there is no positive solution to (5.9) and the solution to (5.2)-(5.5) takes the following form

$$
g(x)=\beta_{1} x+P, \quad x \geq 0 .
$$

The associated optimal strategy is to distribute all of the surplus as dividends and claim the liquidation value immediately.

\subsection{The case with forced financing}

In the case $P<\frac{\beta_{1} \theta_{1} a}{\delta}$ and $0<K<H\left(\varsigma^{*}\right)$, there is no suitable solution to (5.2)-(5.5). This means that it is optimal to raise new capital once the surplus is zero, and bankruptcy is forbidden forever. In this case, the solution $g(x)$ to HJB equations (3.1) and (3.2) should satisfy

$$
\begin{aligned}
& \max _{0 \leq q \leq 1}\left\{\mathscr{A}^{q} g(x)\right\}=0, \quad 0<x<\tilde{d}_{0}, \\
& \beta_{1}-g^{\prime}(x)=0, \quad x \geq \tilde{d}_{0}, \\
& \mathscr{C} g(0)-g(0)=0, \\
& g(0) \geq P,
\end{aligned}
$$


with some parameter $0 \leq \tilde{d}_{0}<\infty$. Let $g(x)$ be given by (5.6), then the solution for (5.17)-(5.20) is $g_{1}(x)=$ $g\left(x+\varrho^{*}\right)$; that is,

$$
g_{1}(x)=\left\{\begin{array}{lc}
\beta_{1}\left(x-\tilde{d}_{0}\right)+g_{1}\left(\tilde{d}_{0}\right), & x \geq \tilde{d}_{0}, \\
l_{1} e^{r_{+}\left(x-\tilde{d}_{0}\right)}+l_{2} e^{r_{-}\left(x-\tilde{d}_{0}\right)}, & 0 \leq x \leq \tilde{d}_{0},
\end{array}\right.
$$

where $\tilde{d}_{0}=d_{0}-\varrho^{*}>0$ and $\varrho^{*} \in\left(0, \varsigma^{*}\right)$ is the unique solution such that

$$
g\left(\varsigma^{*}\right)-g\left(\varrho^{*}\right)-\beta_{2}\left(\varsigma^{*}-\varrho^{*}\right)-K=0 .
$$

Correspondingly, $q^{\pi^{*}}(x) \equiv 1$ is the optimal retention level.

\subsection{The value function and optimal strategy}

In this subsection, we identify the explicit solutions to the value function and construct the associated optimal strategies under condition (5.1).

Theorem 5.1. Under condition (5.1), $V(x)$ and $\pi^{*}$ can be established in the following four cases, which exhaust all of the possibilities. In what follows, $\tilde{d}_{0}=d_{0}-\varrho^{*}$ and $d_{0}, \varsigma^{*}, H\left(\varsigma^{*}\right)$ and $\varrho^{*}$ are defined by $(5.9)$, (5.13), (5.14) and (5.22), respectively.

Case 1: $P<\frac{\beta_{1} \theta_{1} a}{\delta}$ and $\beta_{1}<g^{\prime}(0)=\frac{\beta_{1}}{r_{+}-r_{-}}\left(r_{+} e^{-r_{-} d_{0}}-r_{-} e^{-r_{+} d_{0}}\right) \leq \beta_{2}$

The value function $V(x)$ is identical to $g(x)$ in (5.6). The optimal strategy $\pi^{*}=\left(q^{\pi^{*}}, D^{\pi^{*}}, R^{\pi^{*}}\right)$ satisfies that

$$
\left\{\begin{array}{l}
X_{t}^{\pi^{*}}=x+\theta_{1} a t+b B_{t}-D_{t}^{\pi^{*}} \\
X_{t}^{\pi^{*}} \leq d_{0}
\end{array}\right.
$$

The optimal dividend strategy $D^{\pi^{*}}$ is barrier style, which can be characterised by (4.67), where $u=d_{0}$. Moreover, $R_{t}^{\pi^{*}} \equiv 0$ and $q^{\pi^{*}}(x) \equiv 1$ for all $x \geq 0$.

Case 2: $P<\frac{\beta_{1} \theta_{1} a}{\delta}, g^{\prime}(0)=\frac{\beta_{1}}{r_{+}-r_{-}}\left(r_{+} e^{-r_{-} d_{0}}-r_{-} e^{-r_{+} d_{0}}\right)>\beta_{2}$ and $K \geq H\left(\varsigma^{*}\right)$

The value function $V(x)$ and associated optimal strategy $\pi^{*}$ take the same forms as those in Case 1.

Case 3: $P \geq \frac{\beta_{1} \theta_{1} a}{\delta}$

The solutions to $V(x)$ and $\pi^{*}$ are the same as those in Case 6 of Theorem 4.1.

Case 4: $P<\frac{\beta_{1} \theta_{1} a}{\delta}, g^{\prime}(0)=\frac{\beta_{1}}{r_{+}-r_{-}}\left(r_{+} e^{-r_{-} d_{0}}-r_{-} e^{-r_{+} d_{0}}\right)>\beta_{2}$ and $0<K<H\left(\varsigma^{*}\right)$

The value function $V(x)$ takes the same form as $g_{1}(x)$ in (5.21). The optimal strategy $\pi^{*}=\left(q^{\pi^{*}}, D^{\pi^{*}}, R^{\pi^{*}}\right)$ satisfies that

$$
\left\{\begin{array}{l}
X_{t}^{\pi^{*}}=x+\theta_{1} a t+b B_{t}-D_{t}^{\pi^{*}}+\sum_{n=1}^{\infty} I_{\left\{\tau_{n}^{\pi^{*}} \leq t\right\}} \eta_{n}^{\pi^{*}} ; \\
0 \leq X_{t}^{\pi^{*}} \leq \tilde{d}_{0} .
\end{array}\right.
$$

The optimal reinsurance policy is $q^{\pi^{*}}(x) \equiv 1$. The optimal dividend strategy $D^{\pi^{*}}$ is the barrier style with level $\tilde{d}_{0}=d_{0}-\varrho^{*}>0$, which is described by (4.67), where $u=\tilde{d}_{0}$. It is profitable to finance when and only when the surplus is zero, and the surplus immediately jumps to $\tilde{\varsigma}^{*}=\varsigma^{*}-\varrho^{*}$ once it reaches 0 by issuing equities. Mathematically, $R^{\pi^{*}}$ is characterised by (4.71) and

$$
\eta_{n}^{\pi^{*}} \equiv \tilde{\varsigma}^{*}=\varsigma^{*}-\varrho^{*}, n=1,2, \cdots
$$

Proof. The conclusions can be proven as in Theorem 4.1, thus the details are omitted here.

Remark 5.1. (1) If the cost of reinsurance is relatively high, i.e., (5.1) holds, then the manager does not consider reinsurance. Consequently, Problem 2.1 is simplified to an ordinary optimal dividend and financing control problem. Theorem 5.1 provides the solutions in four cases that explore all of the possibilities.

When no issue of equity is optimal, as in Cases 1-3, the dividend barrier $d_{0}$ is increasing with respect to $\beta_{1}$ in the case of $P \geq 0$; and decreasing in the case of $P<0$. In addition, when $P$ increases on the interval 
$\left(-\infty, \frac{\beta_{1} \theta_{1} a}{\delta}\right)$, the dividend barrier $d_{0}$ gradually decreases. These results come from the property of (5.9). The insurance company declares bankruptcy immediately once the liquidation value $P$ exceeds $\frac{\beta_{1} \theta_{1} a}{\delta}$.

No bankruptcy is optimal if and only if the costs for financing are relative low, i.e., $\beta_{2}<g^{\prime}(0)$ and $K<H\left(\varsigma^{*}\right)$. See Case 4 . The dividend barrier $\tilde{d}_{0}$ and the amount of financing $\varsigma^{*}$ are both increasing with respect to $K$.

(2) If we let $\theta_{2} \rightarrow \theta_{1}$ in the model, the reinsurance reduces to the "cheap" style, then the transaction cost of the reinsurance contract vanishes. Thus, the company can keep the surplus non-negative forever by taking $q \equiv 1$, i.e., transferring all the business to the reinsurer. If the company never declares bankruptcy to claim a negative liquidation value, then the optimisation problem with $P<0$ does not make sense. See, for example, Taksar (2000b), Xu and Zhou (2012), Yao et al. (2014), etc. Obviously, the assumption of "non-cheap" reinsurance seems more reasonable.

(3) From the results of Theorems 4.1 and 5.1, we know that the strategies without financing are optimal when at least one of the two cost factors $K$ and $\beta_{2}$ is large enough, which corresponds to the cases studied in Liang and Young (2012). Similarly, the optimisation problem studied in Peng et al. (2012) can be viewed as a special case in this paper when $P \rightarrow-\infty$. The verification processes are left to the interested readers.

\section{Conclusion}

To maximise an insurance company's value, we consider a combined optimal financing-reinsurance-dividend distribution problem. To reflect reality, the liquidation value at bankruptcy and the transaction costs incurred in the control processes are included in the risk model. We use the stochastic control method to solve the optimisation problem and obtain some interesting results. The insurer should take all risks if the reinsurance is too expensive, and likewise should not choose financing if the costs are too high. The insurer does not distribute dividends until the surplus exceeds some level, and the excess is paid out immediately as dividends. When the liquidation value is too large, it is optimal to distribute all surplus as dividends and claim the liquidation value immediately. The value function can be expressed in terms of retention function $q^{\pi^{*}}(x)$, which is an increasing function of surplus $x \geq 0$. The initial (minimal) retention level $q^{\pi^{*}}(0) \in(\rho, 1]$ is an increasing function of liquidation value $P$. Due to reinsurance contract costs, the insurer's admissible retention must be larger than the lowest level $\rho$ whenever the reinsurance is taken. In summary, the optimal strategies depend on the relationships among the parameters. The main contribution of this paper is that we take both financing and bankruptcy into account and investigate the effects of liquidation value $P$ on optimal strategies, for $P \in \mathbb{R}$. Compared with the work of Liang and Young (2012) and Peng et al. (2012), the risk models and associated results herein are extended and the techniques are different.

Appendix A. The proof of Theorem 3.1

\section{Appendix}

Proof. For each given strategy $\pi=\left(q^{\pi}, D^{\pi}, R^{\pi}\right) \in \Pi$, define $\Lambda_{D}^{\pi}=\left\{s: D_{s-}^{\pi} \neq D_{s}^{\pi}\right\}, \Lambda_{R}^{\pi}=\left\{s: R_{s-}^{\pi} \neq R_{s}^{\pi}\right\}=$ $\left\{\tau_{1}^{\pi}, \tau_{2}^{\pi}, \cdots, \tau_{n}^{\pi}, \cdots\right\}$. Let $\hat{D}_{t}^{\pi}=\sum_{s \in \Lambda_{D}^{\pi}, s \leq t}\left(D_{s}^{\pi}-D_{s-}^{\pi}\right)$ be the discontinuous part of $D_{t}^{\pi}$ and $\tilde{D}_{t}^{\pi}=D_{t}^{\pi}-\hat{D}_{t}^{\pi}$

be the continuous part of $D_{t}^{\pi}$. Similarly, $\hat{R}_{t}^{\pi}$ and $\tilde{R}_{t}^{\pi}$ stand for the discontinuous and continuous parts of $R_{t}^{\pi}$, respectively. Then, applying Itô's formula, we derive that

$$
\begin{aligned}
& e^{-\delta\left(t \wedge T^{\pi}\right)} v\left(X_{t \wedge T^{\pi}}^{\pi}\right)-v(x) \\
& =\int_{0}^{t \wedge T^{\pi}} e^{-\delta s} \mathscr{A}^{q^{\pi}} v\left(X_{s}^{\pi}\right) \mathrm{d} s+\int_{0}^{t \wedge T^{\pi}} b q_{s}^{\pi} \mathrm{d} B_{s}-\int_{0}^{t \wedge T^{\pi}} e^{-\delta s} v^{\prime}\left(X_{s}^{\pi}\right) \mathrm{d} \tilde{D}_{s}^{\pi} \\
& \quad+\int_{0}^{t \wedge T^{\pi}} e^{-\delta s} v^{\prime}\left(X_{s}^{\pi}\right) \mathrm{d} \tilde{R}_{s}^{\pi}+\sum_{s \in \Lambda_{R}^{\pi} \cup \Lambda_{D}^{\pi}, s \leq t \wedge T^{\pi}} e^{-\delta s}\left(v\left(X_{s}^{\pi}\right)-v\left(X_{s-}^{\pi}\right)\right) .
\end{aligned}
$$


The last term on the right side can be written as

$$
\begin{aligned}
& \sum_{s \in \Lambda_{R}^{\pi} \cup \Lambda_{D}^{\pi}, s \leq t \wedge T^{\pi}} e^{-\delta s}\left(v\left(X_{s}^{\pi}\right)-v\left(X_{s-}^{\pi}\right)\right) \\
= & \sum_{s \in \Lambda_{D}^{\pi}, s \leq t \wedge T^{\pi}} e^{-\delta s}\left(v\left(X_{s}^{\pi}\right)-v\left(X_{s-}^{\pi}\right)\right)+\sum_{s \in \Lambda_{R}^{\pi}, s \leq t \wedge T^{\pi}} e^{-\delta s}\left(v\left(X_{s}^{\pi}\right)-v\left(X_{s-}^{\pi}\right)\right) \\
\leq & -\sum_{s \in \Lambda_{D}^{\pi}, s \leq t \wedge T^{\pi}} e^{-\delta s} \beta_{1}\left(D_{s}^{\pi}-D_{s-}^{\pi}\right)+\sum_{n=1}^{\infty} e^{-\delta \tau_{n}^{\pi}}\left(\beta_{2} \eta_{n}^{\pi}+K\right) I_{\left\{\tau_{n}^{\pi} \leq t \wedge T^{\pi}\right\}},
\end{aligned}
$$

where the inequality is because $v(x)$ satisfies the HJB equation (3.1) with $v^{\prime}(x) \geq \beta_{1}$ and $\mathscr{C} v(x) \leq v(x)$. Moreover, given (3.1), the first term on the right side of (A.1) is non-positive. Hence, substituting (A.2) into (A.1), we obtain

$$
\begin{aligned}
e^{-\delta\left(t \wedge T^{\pi}\right)} v\left(X_{t \wedge T^{\pi}}^{\pi}\right) \leq & v(x)+\int_{0}^{t \wedge T^{\pi}} e^{-\delta s} b q_{s}^{\pi} v^{\prime}\left(X_{s}^{\pi}\right) \mathrm{d} B_{s} \\
& -\beta_{1} \int_{0}^{t \wedge T^{\pi}} e^{-\delta s} \mathrm{~d} D_{s}^{\pi}+\sum_{n=1}^{\infty} e^{-\delta \tau_{n}^{\pi}}\left(\beta_{2} \eta_{n}^{\pi}+K\right) I_{\left\{\tau_{n}^{\pi} \leq t \wedge T^{\pi}\right\}} .
\end{aligned}
$$

Given that $v(x)$ is an increasing function and $v(0) \geq P$, we have

$$
\begin{aligned}
e^{-\delta\left(t \wedge T^{\pi}\right)} P \leq & v(x)+\int_{0}^{t \wedge T^{\pi}} e^{-\delta s} b q_{s}^{\pi} v^{\prime}\left(X_{s}^{\pi}\right) \mathrm{d} B_{s} \\
& -\beta_{1} \int_{0}^{t \wedge T^{\pi}} e^{-\delta s} \mathrm{~d} D_{s}^{\pi}+\sum_{n=1}^{\infty} e^{-\delta \tau_{n}^{\pi}}\left(\beta_{2} \eta_{n}^{\pi}+K\right) I_{\left\{\tau_{n}^{\pi} \leq t \wedge T^{\pi}\right\}} .
\end{aligned}
$$

The stochastic integral with respect to the Brownian motion in (A.4) is a uniformly integratable martingale if $v^{\prime}(x)$ is bounded. Taking expectation and limit on both sides of (A.4) yields

$$
v(x) \geq \mathrm{E}_{x}\left(\beta_{1} \int_{0}^{T^{\pi}} e^{-\delta s} \mathrm{~d} D_{s}^{\pi}+P e^{-\delta T^{\pi}}-\sum_{n=1}^{\infty} e^{-\delta \tau_{n}^{\pi}}\left(\beta_{2} \eta_{n}^{\pi}+K\right) I_{\left\{\tau_{n}^{\pi} \leq T^{\pi}\right\}}\right)=V(x ; \pi) .
$$

Consequently, $v(x) \geq V(x)$ follows.

Appendix B. The proof of inequality (4.45)

Proof. In the case $2 \theta_{1} \leq \theta_{2}<\theta_{1}+\sqrt{\theta_{1}^{2}+2 \delta\left(\frac{b}{a}\right)^{2}}$, direct computations show that the left side of (4.45) is non-positive, so it holds automatically. Next, we focus on the opposite case $\theta_{1}<\theta_{2}<2 \theta_{1}$. Let us define a function in $\theta_{2}$ as

$$
\Psi\left(\theta_{2}\right):=\frac{b^{2}}{b^{2}+\frac{\theta_{2} a}{r_{+}}}\left(\frac{b^{2}+\frac{\theta_{2} a}{r_{+}}}{b^{2}+\frac{\theta_{2} a}{r_{-}}}\right)^{\frac{-r_{-}}{r_{+}-r_{-}}}\left(\theta_{1}-\frac{1}{2} \theta_{2}\right), \quad \theta_{2} \in\left[\theta_{1}, 2 \theta_{1}\right) .
$$

Then,

$$
\log \Psi\left(\theta_{2}\right)=\log b^{2}-\log \left(b^{2}+\frac{\theta_{2} a}{r_{+}}\right)-\frac{r_{-}}{r_{+}-r_{-}}\left(\log \left(b^{2}+\frac{\theta_{2} a}{r_{+}}\right)-\log \left(b^{2}+\frac{\theta_{2} a}{r_{-}}\right)\right)+\log \left(\theta_{1}-\frac{1}{2} \theta_{2}\right) .
$$

Vieta's theorem leads to

We deduce that

$$
r_{+}+r_{-}=-\frac{2 \delta}{b^{2}}, \quad r_{+} r_{-}=-\frac{2 \theta_{1} a}{b^{2}}
$$

$$
\begin{aligned}
\frac{\mathrm{d}}{\mathrm{d} \theta_{2}} \log \Psi\left(\theta_{2}\right) & =\frac{a}{\left(b^{2}+\frac{\theta_{2} a}{r_{+}}\right)\left(r_{-}-r_{+}\right)}+\frac{a}{\left(b^{2}+\frac{\theta_{2} a}{r_{-}}\right)\left(r_{+}-r_{-}\right)}-\frac{1}{2\left(\theta_{1}-\frac{1}{2} \theta_{2}\right)} \\
& =\frac{-a^{2} \theta_{2}}{r_{+} r_{-}\left(b^{2}+\frac{\theta_{2} a}{r_{+}}\right)\left(b^{2}+\frac{\theta_{2} a}{r_{-}}\right)}-\frac{1}{2\left(\theta_{1}-\frac{1}{2} \theta_{2}\right)} \\
& =\frac{1}{\frac{2 \delta b^{2}}{\theta_{2} a^{2}}+2\left(\theta_{1}-\frac{1}{2} \theta_{2}\right)}-\frac{1}{2\left(\theta_{1}-\frac{1}{2} \theta_{2}\right)}<0 .
\end{aligned}
$$


Thus, $\Psi\left(\theta_{2}\right)$ is decreasing on the interval $\left[\theta_{1}, 2 \theta_{1}\right)$. With the help of $b^{2}+\frac{\theta_{1} a}{r_{+}}>b^{2}+\frac{\theta_{1} a}{r_{-}}>0$ and $0<\frac{-r_{-}}{r_{+}-r_{-}}<1$, we derive

$$
\begin{aligned}
\Psi\left(\theta_{2}\right) \leq \Psi\left(\theta_{1}\right) & =\frac{b^{2}}{b^{2}+\frac{\theta_{1} a}{r_{+}}}\left(\frac{b^{2}+\frac{\theta_{1} a}{r_{+}}}{b^{2}+\frac{\theta_{1} a}{r_{-}}}\right)^{\frac{-r_{-}}{r_{+}-r_{-}}}\left(\theta_{1}-\frac{1}{2} \theta_{1}\right) \\
& \leq \frac{b^{2}}{2\left(b^{2}+\frac{\theta_{1} a}{r_{-}}\right)} \theta_{1}<\theta_{1} .
\end{aligned}
$$

The last inequality is confirmed by the definition of $r_{-}$, and (4.45) is proved when $\theta_{1}<\theta_{2}<2 \theta_{1}$. In summary, (4.45) holds for all $\theta_{2} \in\left(\theta_{1}, \theta_{1}+\sqrt{\theta_{1}^{2}+2 \delta\left(\frac{b}{a}\right)^{2}}\right)$.

Appendix C. The proof of Case 7 in Theorem 4.1

Proof. Suppose that $P<\frac{k_{3} a}{\delta}\left(\theta_{1}-\frac{1}{2} \theta_{2}\right), f^{\prime}(0)=k_{3} e^{\int_{0}^{x_{0}} \frac{\theta_{2} a}{b^{2} q(z)} \mathrm{d} z}>\beta_{2} \geq k_{3}$ and $K<I\left(\xi_{1}^{*}\right)$. First, we verify that $f_{1}(x)$ and $q_{1}^{\pi^{*}}$ satisfies HJB equations (3.1) and (3.2).

- Step 1: To show $\max _{0 \leq q \leq 1}\left\{\mathscr{A}^{q} f_{1}(x)\right\} \leq 0$ on $[0, \infty)$.

(i) If $0 \leq x \leq u_{1}$, by construction, $f_{1}(x)$ and $q_{1}^{\pi^{*}}(x)$ satisfy $(4.49)$ with $\tilde{u}_{0}=u_{1}$. That is, $\max _{0 \leq q \leq 1}\left\{\mathscr{A}^{q} f_{1}(x)\right\}=$ $\mathscr{A}^{q_{1}^{\pi^{*}}} f_{1}(x)=0$.

(ii) If $x>u_{1}$, then $f_{1}(x) \geq f_{1}\left(u_{1}\right), f_{1}^{\prime}(x)=f_{1}^{\prime}\left(u_{1}\right)=\beta_{1}$ and $f_{1}^{\prime \prime}(x)=f_{1}^{\prime \prime}\left(u_{1}\right)=0$. So, for each $q \in[0,1]$, we derive that

$$
\begin{aligned}
\mathscr{A}^{q} f_{1}(x) & =\frac{1}{2} q^{2} b^{2} f_{1}^{\prime \prime}(x)+\left(\theta_{1}-(1-q) \theta_{2}\right) a f_{1}^{\prime}(x)-\delta f_{1}(x) \\
& =\frac{1}{2} q^{2} b^{2} f_{1}^{\prime \prime}\left(u_{1}\right)+\left(\theta_{1}-(1-q) \theta_{2}\right) a f_{1}^{\prime}\left(u_{1}\right)-\delta f_{1}(x) \\
& \leq \frac{1}{2} q^{2} b^{2} f_{1}^{\prime \prime}\left(u_{1}\right)+\left(\theta_{1}-(1-q) \theta_{2}\right) a f_{1}^{\prime}\left(u_{1}\right)-\delta f_{1}\left(u_{1}\right) \\
& =\mathscr{A}^{q} f_{1}\left(u_{1}\right) \leq 0 .
\end{aligned}
$$

- Step 2: To show $f_{1}^{\prime}(x) \geq \beta_{1}$. It can be established directly from the expression of $f_{1}(x)$ in $(4.53)$.

- Step 3: To show $\mathscr{C} f_{1}(x) \leq f_{1}(x)$. We have

$$
\begin{aligned}
\mathscr{C} f_{1}(x)-f_{1}(x) & =\max _{y \geq 0}\left\{f_{1}(x+y)-\beta_{2} y-K\right\}-f_{1}(x) \\
& =\max _{y \geq 0}\left\{\int_{x}^{x+y}\left(f_{1}^{\prime}(s)-\beta_{2}\right) \mathrm{d} s\right\}-K .
\end{aligned}
$$

(i) If $0 \leq x \leq \eta_{1}^{*}$, then $f_{1}^{\prime}(x)-\beta_{2} \geq 0$ if and only if $0 \leq x \leq \eta_{1}^{*}$. Thus,

$$
\begin{aligned}
\mathscr{C} f_{1}(x)-f_{1}(x) & =\max _{y \geq 0}\left\{\int_{x}^{x+y}\left(f_{1}^{\prime}(s)-\beta_{2}\right) \mathrm{d} s\right\}-K \\
& \leq \int_{0}^{\eta_{1}^{*}}\left(f_{1}^{\prime}(s)-\beta_{2}\right) \mathrm{d} s-K=0,
\end{aligned}
$$

the equality holds if and only if $x=0$ and $y=\eta_{1}^{*}$.

(ii) If $\eta_{1}^{*}<x<\infty$, the inequality $f_{1}^{\prime}(x)-\beta_{2}<0$ is always true, then

$$
\begin{aligned}
\mathscr{C} f_{1}(x)-f_{1}(x) & =\max _{y \geq 0}\left\{\int_{x}^{x+y}\left(f_{1}^{\prime}(s)-\beta_{2}\right) \mathrm{d} s\right\}-K \\
& =-K<0 .
\end{aligned}
$$

- Step 4: Clearly, $f_{1}(x)=f\left(x+p^{*}\right)>P$ is true because $f_{1}^{\prime}(x) \geq \beta_{1}$ and $f_{1}(0)=f\left(p^{*}\right)>f(0)=P$.

Thus, $f_{1}(x)$ satisfies (3.1) and (3.2). Clearly, $f_{1}(x)$ is a twice continuously differentiable with increasing 
and concave function, and its derivative satisfies $\beta_{1} \leq f_{1}^{\prime}(x) \leq f_{1}^{\prime}(0)<\infty$. Thus, $f_{1}(x) \geq V(x)$ holds according to Theorem 3.1. Finally, we verify the optimality of strategy $\pi^{*}=\left(q_{1}^{\pi^{*}}, D^{\pi^{*}}, R^{\pi^{*}}\right) \in \Pi$ as described by (4.54), (4.67), where $u=u_{1},(4.71)$ and (4.72). Given that $\mathscr{A}^{q_{1}^{\pi^{*}}} f_{1}\left(X_{t}^{\pi^{*}}\right)=0$ for $0 \leq X_{t}^{\pi^{*}} \leq u_{1}$, we have

$$
\int_{0}^{t \wedge T^{\pi^{*}}} e^{-\delta s} \mathscr{A}^{q_{1}^{\pi^{*}}} f_{1}\left(X_{s}^{\pi^{*}}\right) \mathrm{d} s=\int_{0}^{t \wedge T^{\pi^{*}}} e^{-\delta s} \mathscr{A}^{q_{1}^{\pi^{*}}} f_{1}\left(X_{s}^{\pi^{*}}\right) I_{\left\{0 \leq X_{s}^{\pi^{*}} \leq u_{1}\right\}} \mathrm{d} s=0 .
$$

Furthermore, (4.67), (4.71) and (4.72) indicate that

$$
\begin{aligned}
& \sum_{s \in \Lambda_{D}^{\pi^{*}} \cup \Lambda_{R}^{\pi^{*}}, s \leq t \wedge T^{\pi^{*}}} e^{-\delta s}\left(f_{1}\left(X_{s}^{\pi^{*}}\right)-f_{1}\left(X_{s-}^{\pi^{*}}\right)\right) \\
= & \sum_{s \in \Lambda_{D}^{\pi^{*}}, s \leq t \wedge T^{\pi^{*}}} e^{-\delta s}\left(f_{1}\left(X_{s}^{\pi^{*}}\right)-f_{1}\left(X_{s-}^{\pi^{*}}\right)\right) I_{\left\{X_{s}^{\pi^{*}}=u_{1}\right\}}+\sum_{s \in \Lambda_{R}^{\pi^{*}}, s \leq t \wedge T^{\pi^{*}}} e^{-\delta s}\left(f_{1}\left(X_{s}^{\pi^{*}}\right)-f_{1}\left(X_{s-}^{\pi^{*}}\right)\right) I_{\left\{X_{s-}^{\pi^{*}}=0\right\}} \\
= & -\sum_{s \in \Lambda_{D}^{\pi^{*}}, s \leq t \wedge T^{\pi^{*}}} e^{-\delta s} \beta_{1}\left(D_{s}^{\pi^{*}}-D_{s-}^{\pi^{*}}\right)+\sum_{n=1}^{\infty} e^{-\delta \tau_{n}^{\pi^{*}}}\left(\beta_{2} \eta_{n}^{\pi^{*}}+K\right) I_{\left\{\tau_{n}^{\pi^{*}} \leq t \wedge T T^{\pi^{*}}\right\}} .
\end{aligned}
$$

Replacing $\pi, T^{\pi}, v$ by $\pi^{*}, T^{\pi^{*}}=\infty, f_{1}$ in Itô's formula (A.1) and taking expectations, we have

$$
f_{1}(x)=\mathrm{E}_{x}\left[e^{-\delta t} f_{1}\left(X_{t}^{\pi^{*}}\right)\right]+\mathrm{E}_{x}\left(\beta_{1} \int_{0}^{t} e^{-\delta s} \mathrm{~d} D_{s}^{\pi^{*}}-\sum_{n=1}^{\infty} e^{-\delta \tau_{n}^{\pi^{*}}}\left(\beta_{2} \eta_{n}^{\pi^{*}}+K\right) I_{\left\{\tau_{n}^{\pi^{*}} \leq t\right\}}\right) .
$$

Letting $t \rightarrow \infty$, the first term on the right side vanishes, then we obtain

$$
f_{1}(x)=\mathrm{E}_{x}\left(\beta_{1} \int_{0}^{\infty} e^{-\delta s} \mathrm{~d} D_{s}^{\pi^{*}}-\sum_{n=1}^{\infty} e^{-\delta \tau_{n}^{\pi^{*}}}\left(\beta_{2} \eta_{n}^{\pi^{*}}+K\right) I_{\left\{\tau_{n}^{\pi^{*}}<\infty\right\}}\right)=V\left(x ; \pi^{*}\right),
$$

which, together with $f_{1}(x) \geq V(x)$, establishes that $f_{1}(x)=V(x)=V\left(x ; \pi^{*}\right)$.

\section{Acknowledgments}

The authors are grateful to the two anonymous referees for their valuable insights and helpful suggestions. This work was supported by the National Natural Science Foundation of China (11571113,11231005,71471081), the Research Grants Council of the Hong Kong Special Administrative Region (project No: HKU 705313P), the Society of Actuaries' Centers of Actuarial Excellence Research Grant, the Humanities and Social Sciences Project of the Ministry Education of China (15YJC910008), the Program of Shanghai Subject Chief Scientist (14XD1401600), the 111 Project (B14019), the Natural Science Foundation of the Jiangsu Higher Education Institutions of China (15KJB110009) and a Project Funded by the Priority Academic Program Development of Jiangsu Higher Education Institutions.

\section{References}

[1] ASMUSSEN, S., H $\phi$ GAARD, B. and TAKSAR, M. (2000) Optimal risk control and dividend distribution policies: example of excess-of-loss reinsurance for an insurance corporation. Finance and Stochastics, 4(3), 299-324.

[2] BAI, L., GUO, J. and ZHANG, H. (2010) Optimal excess-of-loss reinsurance and dividend payments with both transaction costs and taxes. Quantitative Finance, 10(10), 1163-1172.

[3] BARTH, A. and MORENO-BROMBERG, S. (2014) Optimal risk and liquidity management with costly refinancing opportunities. Insurance: Mathematics and Economics, 57, 31-45.

[4] CADEnillas, A., CHOUlli, T., TAKSAR, M. and ZHANG, L. (2006) Classical and impulse stochastic control for the optimization of the dividend and risk policies of an insurance firm. Mathematical Finance, 16(1), 181-202.

[5] CHEN, M., PENG, X. and GUO, J. (2013) Optimal dividend problem with a nonlinear regular-singular stochastic control. Insurance: Mathematics and Economics, 52(3), 448-456.

[6] CHOUlLI, T., TAKSAR, M. and ZHOU, X. (2003) A diffusion model for optimal dividend distribution for a company with constraints on risk control. SIAM Journal on Control and Optimization, 41(6), 1946-1979.

[7] FLeming, W. and SONER, H. (1993) Controlled Markov Process and Viscosity Solutions. London: Springer-Verlag.

[8] GRANDELL, J. (1991) Aspects of Risk Theory. London: Springer-Verlag.

[9] GUAN, H. and LIANG, Z. (2014) Viscosity solution and impulse control of the diffusion model with reinsurance and fixed transaction costs. Insurance: Mathematics and Economics, 54, 109-122.

[10] HE, L. and LIANG, Z. (2009) Optimal financing and dividend control of the insurance company with fixed and proportional transaction costs. Insurance: Mathematics and Economics, 42(3), 88-94. 
[11] H $\phi$ GAARD, B. and TAKSAR, M. (1999) Controlling risk exposure and dividends payout schemes: Insurance company example. Mathematical Finance, 9(2), 153-182.

[12] H $\phi$ GAARD, B. and TAKSAR, M. (2004) Optimal dynamic portfolio selection for a corporation with controllable risk and dividend distribution policy. Quantitative Finance, 9(2), 153-182.

[13] LIANG, Z. and YOUNG, V. (2012) Dividends and reinsurance under a penalty for ruin. Insurance: Mathematics and Economics, 50(3), 437-445.

[14] LIU, W. and HU, Y. (2014) Optimal financing and dividend control of the insurance company with excess-of-loss reinsurance policy. Statistics and Probability Letters, 84, 121-130.

[15] L $\phi K K A$, A. and ZERVOS, M. (2008) Optimal dividend and issuance of equity policies in the presence of proportional costs. Insurance: Mathematics and Economics, 42(3), 954-961.

[16] MENG, H. and SIU, T. (2011) On optimal reinsurance, dividend and reinvestment strategies. Economic Modelling, 28, 211-218.

[17] MNIF, M. and SULEM, M. (2005) Optimal risk control and dividend policies under excess of loss reinsurance. Stochastics, $77(5), 455-476$.

[18] PENG, X., CHEN, M. and GUO, J. (2012) Optimal dividend and equity issuance problem with proportional and fixed transaction costs. Insurance: Mathematics and Economics, 51(3), 576-585.

[19] TAKSAR, M. (2000a) Optimal risk and dividend distribution control models for an insurance company. Mathematical Methods of Operations Research, 51(1), 1-42.

[20] TAKSAR, M. (2000b) Dependence of the optimal risk control decisions on the terminal value for a financial corporation. Annals of Operations Research, 98(1), 89-99.

[21] TAKSAR, M. and HUNDERUP, C. (2007) The influence of bankruptcy value on optimal risk control for diffusion models with proportional reinsurance. Insurance: Mathematics and Economics, 40, 311-321.

[22] TAKSAR, M. and ZHOU, X. (1998) Optimal risk and dividend control for a company with a debt liability. Insurance: Mathematics and Economics, 22, 105-122.

[23] XU, J. and ZHOU, M. (2012) Optimal risk control and dividend distribution policies for a diffusion model with terminal value. Mathematical and Computer Modelling, 56, 180-190.

[24] YAO, D., YANG, H. and WANG, R. (2014) Optimal risk and dividend control problem with fixed costs and salvage value: Variance premium principle. Economic Modelling, 37, 53-64.

[25] ZHOU, M. and YUEN, K.C. (2012) Optimal reinsurance and dividend for a diffusion model with capital injection: Variance premium principle. Economic Modelling, 29, 198-207. 